\title{
Dynamic Method of Stiffness Identification in Impacting Systems for Percussive Drilling Applications
}

\author{
Maolin Liao ${ }^{\mathrm{a}}$, James Ing ${ }^{\mathrm{a}}$, Mukthar Sayah ${ }^{\mathrm{a}}$, Marian Wiercigroch $^{\mathrm{a}}$ \\ ${ }^{a}$ Centre for Applied Dynamics Research, School of Engineering, University of Aberdeen, Aberdeen AB24 3UE, UK
}

\begin{abstract}
This paper introduces a dynamic method for the stiffness identification of an impacted object via analysis of its corresponding impact duration. To accurately detect the impact durations from experimental signals, nonlinear time series methods are applied. Two low-dimensional dynamical systems, including a piecewise-linear impact oscillator and a rock impacting system, are studied experimentally and numerically to demonstrate the proposed method. Meanwhile, the analytical prediction of the impact duration for the period-one one-impact motion is developed. The results of both systems indicate that, for a certain stiffness, the impact duration of the period-one one-impact motion is nearly constant. The higher the stiffness, the lower the impact duration. This monotone correlation provides a mechanism to estimate the stiffness of the impacted object once the impact duration has been accurately detected. The developed method can be used to optimise percussive drilling parameters.
\end{abstract}

Keywords: Stiffness identification; Nonlinear time series analysis; Tangent vector analysis; Impact oscillator; Rock impacting system

\section{Introduction}

Impact is the main mechanism for generating rate of penetration (ROP) in many published percussive drilling applications. Percussive drilling, in contrast to conventional rotary drilling, mainly relies on the accumulated kinetic energy during the falling process to force a drillbit into rocks and form a crushed zone beneath the contacting surface. This mechanism provides two advantages when compared with rotary drilling. Firstly, its kinetic impact load is much higher than the load generally achieved in rotary drilling; hence, percussive drilling can be more efficient when drilling hard rocks. Secondly, for percussive drilling, after an impact, the drillbit rebounds immediately, separating the drillbit from rock until the next impact. Statistically, the contact duration in percussive drilling is $2 \%$ of the total operational duration, which is substantially less than that in rotary drilling [1]. The shorter the contact duration, the less abrasive wear of drillbit. However, pure percussive drilling has been proved to have a low efficiency in actual drilling applications; since repeated impacts can not further crush the impact zone, but compact the rock fragments if they are not removed in time. Under such circumstances, percussion is naturally considered to co-operate with rotation, i.e., rotary-percussive drilling. Rotary-percussive drilling is expected to combine the advantages of both drilling methods, to further stimulate the potential of drilling efficiency. Specifically, the percussion builds a crushed zone beneath the impact area, and then the rotation cuts and shears the fractures initiated by the percussion, simultaneously removing the rock fragments.

Email addresses: m.liao@abdn.ac.uk (Maolin Liao), j.ing@abdn.ac.uk (James Ing), m.sayah@abdn.ac.uk (Mukthar Sayah), m.wiercigroch@abdn.ac.uk (Marian Wiercigroch) 
The main purpose of developing the rotary-percussive drilling is to improve drillbit performance and increase ROP $[2,3,4]$. Hustrulid and Fairhurst comprehensively studied the percussive drilling of rocks, including the basic theory of percussive drilling [5], the force-penetration relationship of rock, and the specific energy determination [6]. They also carried out experiments to verify their theories [7], and mentioned the applications of their model in actual rock drilling [8]. Subsequently, the energy transfer in percussive rock destruction was considered by Lundberg, who also compared the efficiencies of different percussive processes $[9,10,11]$. Powell et al. [12] performed a field experiment, where they tested a percussion drilling system combined with different hybrid polycrystalline diamond compact (PDC) bits, showing the ROP increased over $100 \%$. The latest progress of the rotary-percussive drilling comes from the Resonance Enhanced Drilling (RED) project [13, 14], which is also the motivation of the present work. RED aims to superimpose axial drillbit vibrations upon normal rotary drilling to optimise ROP. This is done by means of a controlled resonance in the borehole. However, due to the heterogeneity and anisotropy of formations, the parameters of axial vibration need to be adjusted so that the resonance conditions can be maintained. This requires a real-time monitoring of the properties of the drilled formation, which is a key aspect within the RED technology. Logging-while-drilling (LWD) [15] is a mature technique for monitoring the drilled formation in real-time. Its measurements include natural gamma ray, neutron porosity, resistivity, sonic speed, nuclear magnetic resonance, and seismic while drilling, etc. Among the variety of the logging measurements, the dynamic responses of the bottom-hole-assembly (BHA) do not attract sufficient attention, although they are beneficial for formation evaluation. The main reason is the limitation of data transmission rates. A large volume of real-time data are necessary for the analysis of the BHA dynamics; however, the data transmission rate of mud-pulse telemetry (MPT) [16] is relatively low, typically as low as 1.5 3.0 bits per second. For electromagnetic telemetry (EMT), data transmission rates of up to 10.0 bits per second can be achieved; however, EMT falls short when drilling exceptionally deep wells, and the signal decays rapidly in certain types of formations, both conditions can cause EMT occasionally to lose the ability of data transmission [17]. As the development of the data transmission techniques, reliable and high-speed telemetry methods make the transmission of a large volume of realtime dynamic data possible. Especially, the newly developed wired drill pipe telemetry [18, 19], whose data transmission rates can be upwards of one megabit per second, can provide more than sufficient data support for dynamic analysis. The ideal condition for RED is to apply the collected dynamic response of the drillbit to identify the stiffness of the drilled rock. This stiffness depends primarily on the in-situ elastic modulus of the rock and the contact area of the drillbit.

The drillbit-rock axial interaction can be modeled by an intermittent impact system, e.g., a harmonically forced impact oscillator interacting with an elastic constraint. Many previous investigations have revealed a rich bifurcation structure for this or similar systems [20, 21, 22]. Okolewska et al. [23] formulated the energy dissipation during impacts and mentioned the method to calculate the impact duration according to the natural period of the oscillator-base system. Pavlovskaia and Wiercigroch [24] separated the oscillation from drift by using relative displacements, based on which Páez Chávez et al. [25] analysed its bifurcations using a computational package TC-HAT [26]. In order to simulate the mechanical characteristics of rock deformation under intermittent contacts, Ajibose et al. used a solid mechanics approach to model the loading and unloading phases of rock contact [27], and demonstrated the correlation between contact force and indentation via quasi-static rock deformation tests [28].

Time series methods are applied in this paper since only limited measurements can be obtained during experiments. Essentially, time series analysis is a sort of data expansion, under which the characteristic parameters of a system can be explored. Nonlinear time series analysis comprises a set of methods to 
extract dynamic information through reconstructing the full dynamics of a system from a single time series [29]. The basic idea behind this method is phase space reconstruction, the purpose of which is to expand a certain variable to a multivariate state space as a representative of the original system. Nonlinear time series analysis was first proposed by Packard et al. [30] and then formalized by Takens [31]. The fundamental theories and techniques of nonlinear time series analysis have been introduced by Kantz and Schreiber in their book [32], and they also developed a software package TISEAN [33] to fulfil the functions mentioned in their book. The standard strategy for phase space reconstruction is delay coordinate embedding; a number of methods have been developed to estimate the embedding parameters. The first minimum of the average mutual information [34] is often used to determine the time delay. Kennel et al. [35] suggested the false nearest neighbour analysis to determine the minimal embedding dimension. Invariant quantities of a system, such as Lyapunov exponents [36], fractal dimension [37] can be calculated, to understand the system state. Recurrence plots [38] are used to detect determinism and stationarity, due to their advantage of intuitively displaying all correlations in a two-dimensional plot.

This paper is organized as follows. Section 2 introduces impact duration detection using nonlinear time series analysis and tangent vector analysis. The modulus and direction gradient of the reconstructed tangent vector are defined to recognise impacts. Section 3 discusses an analytical prediction of the impact duration of the period-one one-impact motion based on the 1-DOF impact oscillator. The corresponding numerical simulation and experiment verify the analytical prediction. The correlation between the stiffness of an impacted beam and the impact duration is explored. In Section 4, a rock impacting system which has $2 \frac{1}{2}$-DOF [39] is studied. Analytical predictions, numerical simulations and experiments are discussed. The loading and unloading stiffnesses of impacted rocks are estimated according to the detected loading and unloading durations, and the relative estimation errors are analysed subsequently. Concluding remarks are provided in Section 5.

\section{Impact duration detection}

The key point of the impact duration detection is to distinguish the impact motion from the nonimpact motion. Especially, when an impact is weak, or a collected signal is noisy, the impact recognition becomes challenging. In this section, nonlinear time series analysis and tangent vector analysis will be combined to detect impact duration from acceleration time series.

\subsection{Nonlinear time series analysis}

A typical nonlinear time series analysis is presented in Fig. 2.1. Its subplot (a) shows the targeted time series, which is the acceleration of the oscillator for the 1-DOF impact oscillator system detailed in Appendix A. The nonlinear time series analysis was carried out using the software package TISEAN [33], this analysis has three main steps.

Step 1: A proper time delay $(d)$ is estimated by the mutual information test. Mutual information $(M I)$ describes the dependence of two variables. For a time series, $M I$ varies as the change of $d$. If $d$ is small, the delayed coordinates are indistinguishable, and the reconstructed trajectory overlaps on the antidiagonal. If $d$ is large, the reconstructed trajectory is overstretched, and loses its intrinsic structure. Basically, the first local minimum of $M I$ corresponds to the proper time delay since overlap and overstretch are avoided simultaneously. For the time series displayed in Fig. 2.1 (a), the obtained proper time delay $d=23$ is shown in Fig. 2.1 (b). Here, the value of $d$ is a multiple of the time step.

Step 2: A sufficient embedding dimension $(m)$ is determined via the false nearest neighbour test. Based on the confirmed time delay $(d)$, the false nearest neighbour test searches for points which are 
(a)

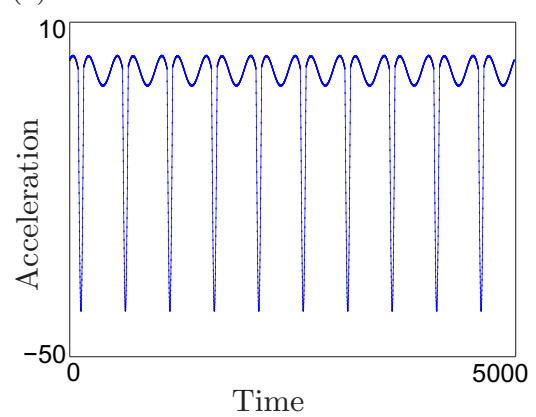

(b)

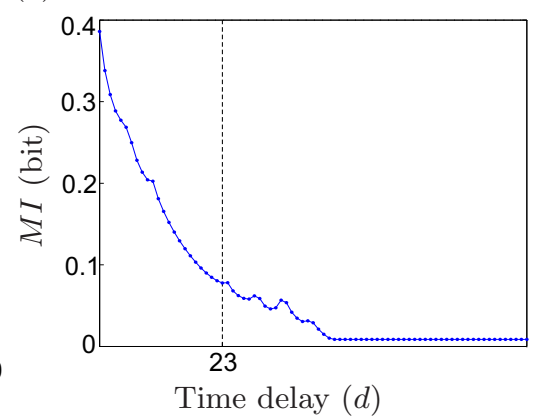

(c)

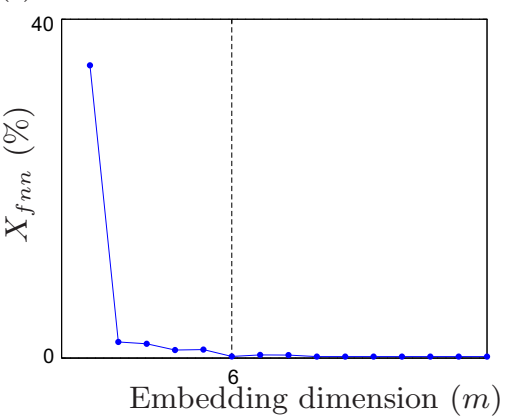

Figure 2.1: Nonlinear time series analysis of impacting motion. Subplot (a) shows an acceleration time series of the oscillator detailed in Appendix A. The time history was simulated with the parameters $a=5.6, e=2.1, \xi=0.01$, $\varphi_{0}=4.86, \beta=18.27, \omega=0.85$ and $\omega_{n}=60.41$. Subplot (b) shows the result of mutual information test $d=23$, subplot (c) shows the result of false nearest neighbour test $m=6$.

neighbours in the current phase space, but will not be neighbours due to further dimension evolution. Specifically, pick the closest neighbour of each point in n-dimension phase space, and compute the ratio of the distances between the corresponding two points in $(\mathrm{n}+1)$-dimension phase space and in $\mathrm{n}$-dimension phase space. If the ratio is larger than a pre-set threshold $(\sigma)$, i.e., the two points move away from each other in a higher dimensional phase space, then they are neighbours only in projection. The result of the false nearest neighbour test for the studied case is shown in Fig. 2.1 (c). $m=6$ is marked as the minimal embedding dimension since its integral ratio of the false nearest neighbours $X_{f n n}=0$; hence a sufficient embedding dimension is any $m \geq 6$, this is because once the attractor is unfold, its structure will be maintained in higher dimensional spaces. Under such circumstances, $m=7$ is chosen as the embedding dimension for the following phase space reconstruction. Strictly speaking, the disappearance of all the false nearest neighbours indicates the minimal embedding dimension. However, for the collected experimental signals, due to the presence of noise, the calculated embedding dimension is larger than its actual minimum, since the noisy time series need extra dimensions to fully unfold. Under such circumstances, the embedding dimension corresponding to the percentage of the false nearest neighbours $\left(X_{f n n}\right)$ which is less than a noise threshold $(\gamma)$ can be accepted as a sufficient embedding dimension. Such a condition will be observed in the following analysis of the time series collected experimentally.

Step 3: The phase space is reconstructed according to the optimised embedding parameters $(d, m)$. Generally, before the phase space reconstruction, the determinism and stationarity of the studied time series should be tested. However, the time series analysed in this subsection comes from the numerical simulation of a confirmed system; hence, its determinism and stationarity have already been guaranteed. The basic procedure of the phase space reconstruction is to transform a time series $\left(a_{1}, a_{2}, \ldots, a_{n-1}\right.$, $\left.a_{n}\right)$ to a group of embedding vectors $\left(\left[a_{1}, a_{1+d}, \ldots, a_{1+(m-1) d}\right], \ldots,\left[a_{i}, a_{i+d}, \ldots, a_{i+(m-1) d}\right], \ldots\right.$, $\left.\left[a_{(n-1)-(m-1) d}, a_{(n-1)-(m-2) d}, a_{(n-1)}\right],\left[a_{n-(m-1) d}, a_{n-(m-2) d}, \ldots, a_{n}\right]\right)$. The time delay, $d$, determines the interval between two successive dimensions in each reconstructed vector; while embedding dimension, $m$, determines the dimension of each reconstructed vector. In order to display the reconstructed phase space properly, three dimensions are chosen from the reconstructed vectors to form a $3 \mathrm{D}$ phase portrait. Different dimension combinations need to be compared, the optimised combination should make the phase portrait unfold, and make the characteristics of each dimension unsheltered. The reconstructed 3D phase portrait for this studied case is shown in Fig. 2.2 (a) using the combination of the 1st, 4th, and 7 th dimensions, i.e., $\left[a_{i}, a_{i+3 d}, a_{i+6 d}\right]$, where $i=1,2, \ldots, n-6 d$. 


\subsection{Tangent vector analysis}

(a)

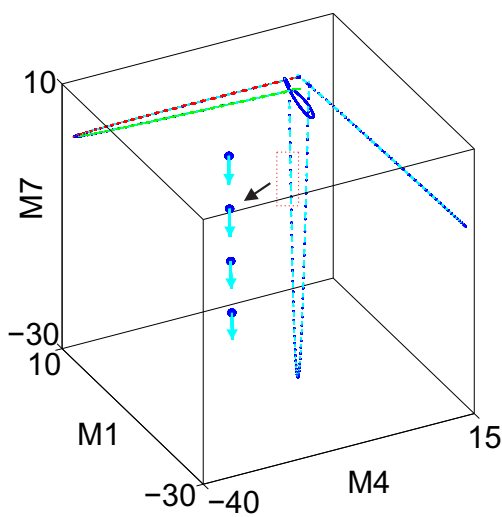

(c)

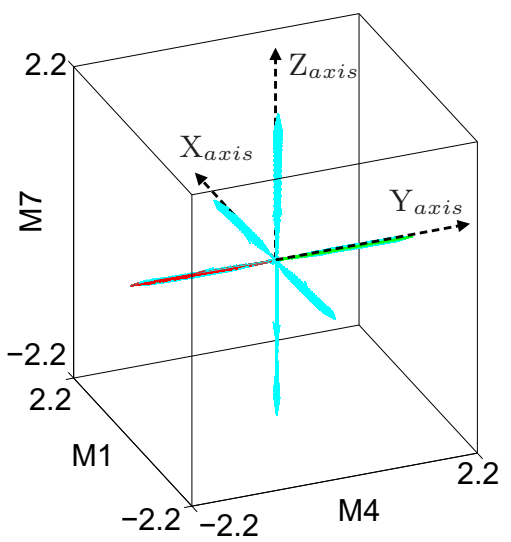

(b)

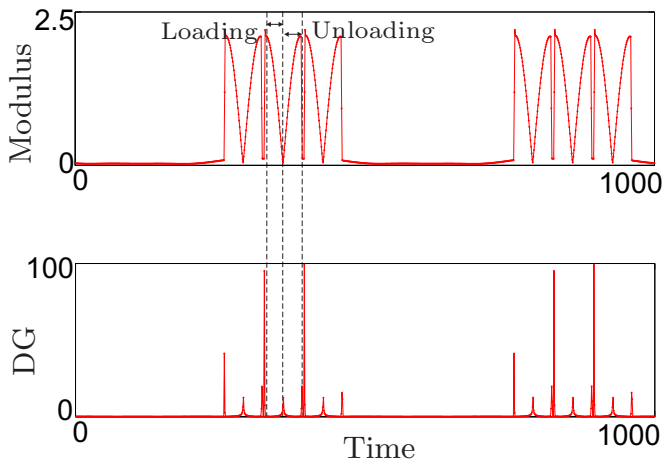

(d)

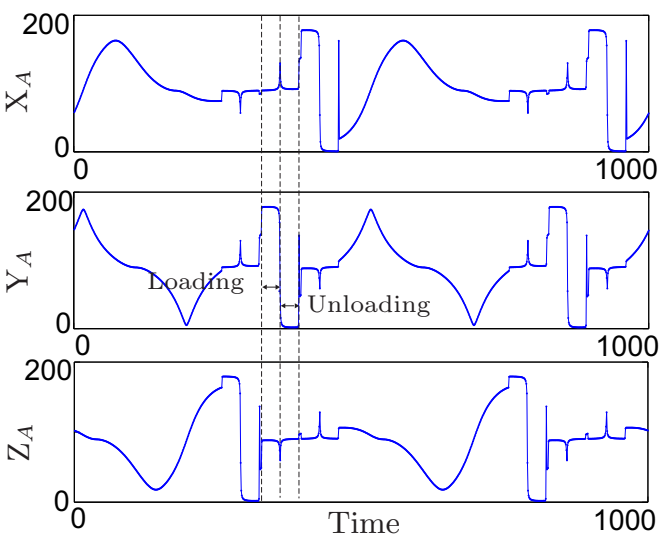

Figure 2.2: (colour online) Tangent vector analysis of impacting motion. (a) The reconstructed 3D (1st, 4th, and 7th dimensions) phase portrait for the time series shown in Fig. 2.1 (a) with embedding parameters $d=23$ and $m=7$. Specifically, the dark blue points constitute the 3D phase portrait, the light blue arrows show the tangent vectors. The part of trajectory marked by a dashed red rectangle is enlarged on its left side to show the tangent vectors clearly. The marked red and green tangent vectors indicate the loading and unloading phases in the 4th dimension. Subplot (b) shows the time series of modulus in the upper plot and the time series of direction gradient in the lower plot. Their peaks indicate impacts. The tangent vectors depicted in subplot (a) are rearranged at the origin in subplot (c). For each tangent vector, three angles with respect to $\mathrm{X}_{a x i s}, \mathrm{Y}_{a x i s}, \mathrm{Z}_{a x i s}$ are calculated, and the obtained angle time series are displayed in subplot (d).

When an impact occurs, it is accompanied by a resistance from the impacted constraint, which triggers an extra increment of the acceleration of the oscillator; hence, an acceleration jump in the time series is expected. However, if the impact is weak, the discontinuity of the acceleration can not be clearly observed. In order to highlight weak impacts, the rate of change of acceleration was taken into consideration, it can be expressed by the modulus of the tangent vectors of the reconstructed 3D phase portrait. In order to get the tangent vectors, linear fitting was utilised to get the slope along the reconstructed trajectory in each dimension. At the $i$ th point, the obtained three slopes $\left(s_{i}, s_{i+3 d}, s_{i+6 d}\right)$, which belong to the 1 st, 4 th, and 7th dimensions, respectively, constitute its tangent vector $T V(i)$. The modulus of this tangent vector, hence, can be calculated as:

$$
\operatorname{Modulus}(i)=\|T V(i)\|=\sqrt{s_{i}^{2}+s_{i+3 d}^{2}+s_{i+6 d}^{2}},
$$


where $i=1,2, \ldots, n-6 d$. The obtained time series of the modulus is shown in the upper plot of (b) in Fig. 2.2. The peaks of this time series correspond to the acceleration jumps in the original time series.

In addition to the modulus, the direction of the tangent vector displays another heuristic character by which to indicate the impacts. In order to show this relationship clearly, all tangent vectors depicted in Fig. 2.2 (a) are rearranged into a bundle initiating at the origin, see Fig. 2.2 (c). All the rearranged tangent vectors can be divided into three groups, which belong to the 1st, 4th, 7th dimensions, respectively. Each group includes a pair of clusters whose orientations are opposite; they represent the loading and unloading phases of an impact. For the non-impact tangent vectors, they gather around the origin since they are quite short compared with impact tangent vectors. For the sake of a precise description of the directions of $3 \mathrm{D}$ tangent vectors, three angles between each tangent vector and three axes, $\mathrm{X}_{\text {axis }}, \mathrm{Y}_{\text {axis }}, \mathrm{Z}_{\text {axis }}$, are calculated; hence, the tangent vector's direction can be defined by the combination of the three angles. The coordinates of the three axes are set as:

$$
X_{\text {axis }}=[1,0,0], \quad Y_{\text {axis }}=[0,1,0], \quad Z_{\text {axis }}=[0,0,1] .
$$

The angles of the $i$ th tangent vector can be calculated in a standard way with respect to the basis vectors as:

$$
\left\{\begin{array}{l}
X_{A}(i)=\arccos \left(\frac{X_{\text {axis }} \cdot T V(i)}{\left\|X_{\text {axis }}\right\|\|T V(i)\|}\right) \frac{180}{\pi} \\
Y_{A}(i)=\arccos \left(\frac{Y_{\text {axis }} \cdot T V(i)}{\left\|Y_{\text {axis }}\right\|\|T V(i)\|}\right) \frac{180}{\pi} \\
Z_{A}(i)=\arccos \left(\frac{Z_{\text {axis }} \cdot T V(i)}{\left\|Z_{\text {axis }}\right\|\|T V(i)\|}\right) \frac{180}{\pi}
\end{array}\right.
$$

where $\cdot$ means dot product, $\|\cdot\|$ stands for modulus. The ranges of the three angles are $\left[0,180^{\circ}\right]$.

The time series of the angles are listed in Fig. 2.2 (d). A series of angle jumps can be observed in these time series, they correspond to the loading and unloading impacts. The same as the rate of change of acceleration (modulus), the rate of angle change of the 3D tangent vector is considered; hence, a new parameter named direction gradient is defined. The direction gradient of the $i$ th tangent vector, $D G(i)$, is written as:

$$
D G(i)=\sqrt{\left(X_{A}(i)-X_{A}(i-1)\right)^{2}+\left(Y_{A}(i)-Y_{A}(i-1)\right)^{2}+\left(Z_{A}(i)-Z_{A}(i-1)\right)^{2}},
$$

where $i=2,3, \ldots, n-(m-1) d$. The obtained time series of the direction gradient is shown in the lower plot of (b) in Fig. 2.2. The peaks of its time series correspond to the positions for the start of loading impact, the loading unloading transferring point, and the end of unloading impact in the original time series; since, in these positions, the angles of the tangent vectors are changed dramatically due to impacts. Therefore, the combination of these two parameters, Modulus $(i)$ and $D G(i)$, provides a robust method for determining impacting positions even for weak impacts.

\subsection{Impact duration estimation}

On the basis of the analysis processed in subsection 2.2, two new parameters, modulus and direction gradient of the reconstructed 3D tangent vector have been defined. Three groups of peaks per period are observed in both time series shown in Fig. 2.2 (b), since the 3D tangent vector is constituted by the 1st, 4th, and 7th dimensions. Any pair of peaks can be used to mark the impact since the peaks will be restored to the same positions in the original time series. The pair of peaks for the 4th dimension are marked by three dashed lines in Fig. 2.2 (b), the start, transferring, and end positions of an impact are, 
therefore, determined. Copy the three dashed lines to Fig. 2.2 (d), the combinations of the angle ranges $\left(\mathrm{X}_{A}, \mathrm{Y}_{A}\right.$, and $\left.\mathrm{Z}_{A}\right)$ for the loading and unloading impacts are confirmed. In Fig. 2.2 (a) and (c), any tangent vector satisfying the loading angle combination is marked as red, or is marked as green if the unloading angle combination is satisfied; since, for periodic motions, all impacts should own the same angle ranges in the reconstructed phase space.

(a)
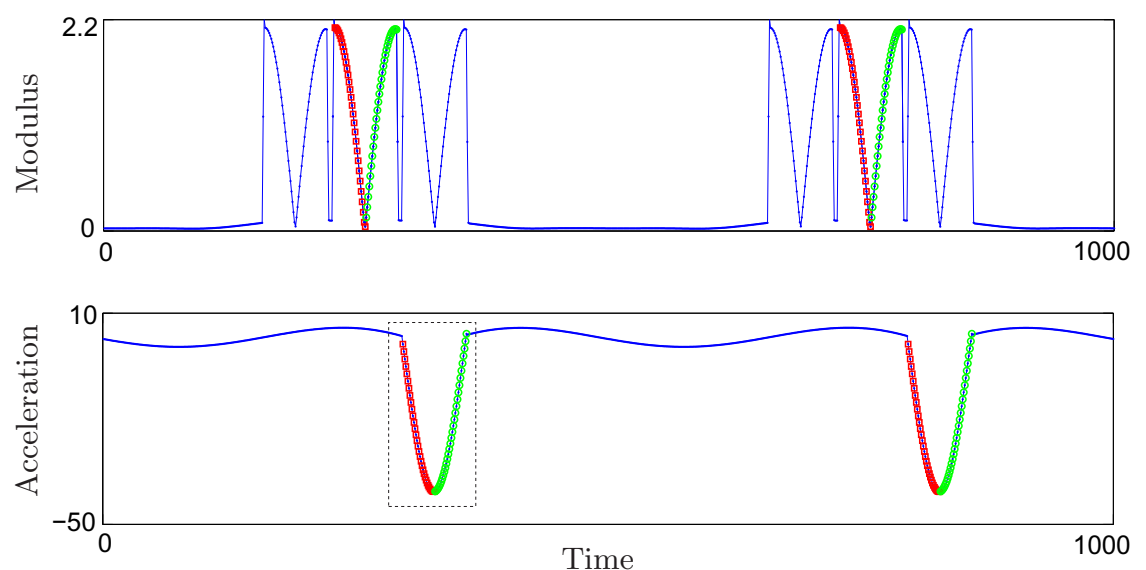

(b)

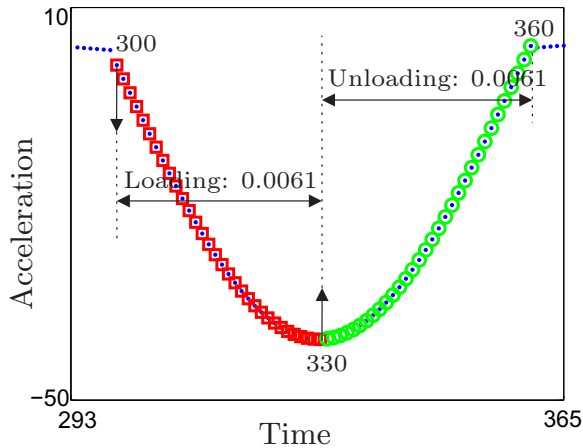

(c)

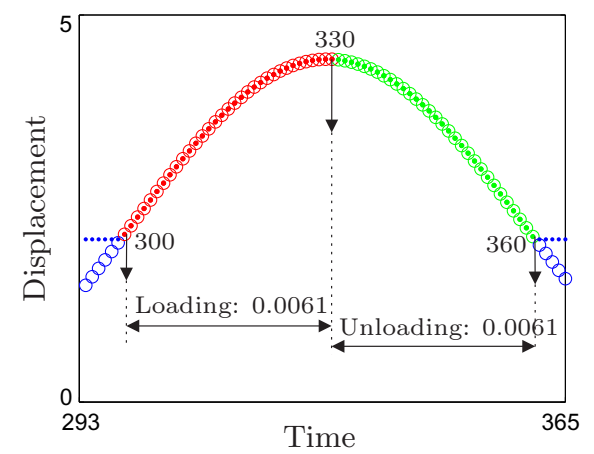

Figure 2.3: (colour online) Impact detection and verification. (a) The upper plot shows the time series of the modulus of the $3 \mathrm{D}$ tangent vectors. The added red and green points represent the loading and unloading impacts in the 4th dimension. The lower plot shows the original acceleration time series, the red and green points are restored from the corresponding points in the upper plot. Subplot (b) is a blow-up of the segment surrounded by a dashed rectangle in subplot (a). In this blow-up, the positions for the start of loading impact (300), loading-unloading transferring point (330), and end of unloading impact (360) are marked. For this impact period, 61 sampling points (300 360) are obtained, according to the step size $0.0002 \mathrm{~s}$, the impact duration is $0.0122 \mathrm{~s}$. Both the loading and unloading durations are $0.0061 \mathrm{~s}$ since they share the loading-unloading transferring point. Subplot (c) shows the displacement of the oscillator (no contact: blue circles, loading: red circles, unloading: green circles) and the displacement of the elastic beam (no contact: blue dots, loading: red dots, unloading: green dots) for the same segment as subplot (b). The intersection of these two displacements means impact.

Through the above analysis, the tangent vectors for the loading and unloading impact phases in the reconstructed phase space have been confirmed (see the upper plot of (a) in Fig. 2.3). The next step is to restore the impact positions to the original time series. This is an inverse operation of the phase space reconstruction. In the reconstructed phase portrait, the points belonging to the 1st dimension keep the same positions as they are in the original time series; while for the points distributed to other dimensions, their original positions need to be calculated according to the equation:

$$
p=i+(j-1) d
$$


where $i, j$ are the index of the tangent vector and the index of the dimension, and $p$ is the corresponding position in the original time series. After the inverse operation, the impacts in the original time series are explored and marked in the lower plot of (a) in Fig. 2.3. In order to verify the detected impacts, there is a blow-up of the segment surrounded by a dashed rectangle in the lower plot of (a) shown in subplot (b). Its impact positions and impact durations are marked in detail. Meanwhile, the displacement of the oscillator and the displacement of the elastic beam for the corresponding segment are simulated and shown in subplot (c), since their meeting means an impact occurring. Comparing subplots (b) and (c), their impact positions and impact durations are in agreement, which verifies this method.

\section{Stiffness identification of the 1-DOF impact oscillator}

For soft impact oscillators, the stiffer the impacted object, the shorter the impact duration. Based on this, a 1-DOF impact oscillator interacting with a linear elastic constraint is studied to explore the stiffness-impact duration correlation.

\subsection{Analytical prediction of impact duration}

Based on the well-studied 1-DOF impact oscillator detailed in Appendix A, numerical continuation of frequency ratio was performed, the details of this continuation using TC-HAT are introduced in [40]. The obtained result is shown in Fig. 3.1 (a), the blue, green, black, and red solid segments reflect the variations of the impact duration for stable period-one non-impact motion, grazing induced period-one one-impact motion, stable period-one two-impact motion and stable period-one one-impact motion, respectively. There is no impact for the blue segment, hence its impact duration maintains zero. The solid green segment indicates the condition near grazing, where the rapid evolution of the attractor happens. For the solid black segment, an increase of the impact duration as increasing frequency ratio can be observed; while the red solid segment has a near constant impact duration.

Considering the stable period-one one-impact motion marked as the red segment, each impact has a pair of loading and unloading phases, during which kinetic energy is converted to elastic strain energy and vice versa. For this 1-DOF impact oscillator, the loading stiffness $k_{l}$ and the unloading stiffness $k_{u}$ of its linear elastic beam are the same, i.e. $k_{l}=k_{u}=k_{2}$; hence, there is no energy dissipation during impact, and $\overrightarrow{v_{\text {in }}}=-\overrightarrow{v_{\text {out }}}$ as shown in Fig. 3.1 (b). From a geometric point of view, the pair of loading and unloading phases can be approximated as half of one period of a simple harmonic motion, providing some additional conditions are met.

Subsequently, the question would be how to define these additional conditions to ensure the assumption is satisfied? According to the analytical solution of the 1-DOF impact oscillator provided by Ing [41], the displacement of the oscillator during the impact phase is expressed as:

$$
x_{\tau}=\frac{e \beta}{1+\beta}+D_{2} \sin \left(\omega \tau+\varphi_{2}\right)+\exp \left(-\xi\left(\tau-\tau_{n}\right)\right)\left(A_{2} \cos \left(\gamma_{2}\left(\tau-\tau_{n}\right)\right)+B_{2} \sin \left(\gamma_{2}\left(\tau-\tau_{n}\right)\right)\right),
$$

where $D_{2}, \gamma_{2}, \varphi_{2}$ are the constants determined by the nondimensionalized parameters, $\xi, \beta$, $\omega$; while $A_{2}$, $B_{2}$ are the constants determined by both the initial conditions $\left(x_{n}, v_{n}\right)$ at $\tau_{n}$, and the nondimensionalized parameters, $e, \xi, \beta, \omega[41]$. In this formulation, $\tau_{n}$ denotes the start of the contact phase. Through a basic trigonometric transform, Eq. 3.1 can be simplified as:

$$
x_{\tau}=\frac{e \beta}{1+\beta}+D_{2} \sin \left(\omega \tau+\varphi_{2}\right)+Q_{2} \sin \left(\gamma_{2} \tau+\phi_{2}\right),
$$


(a)

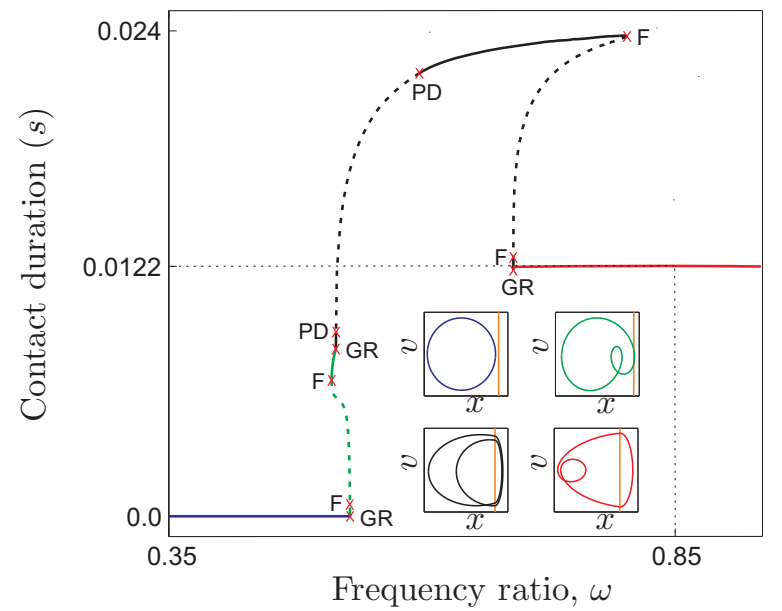

(b)

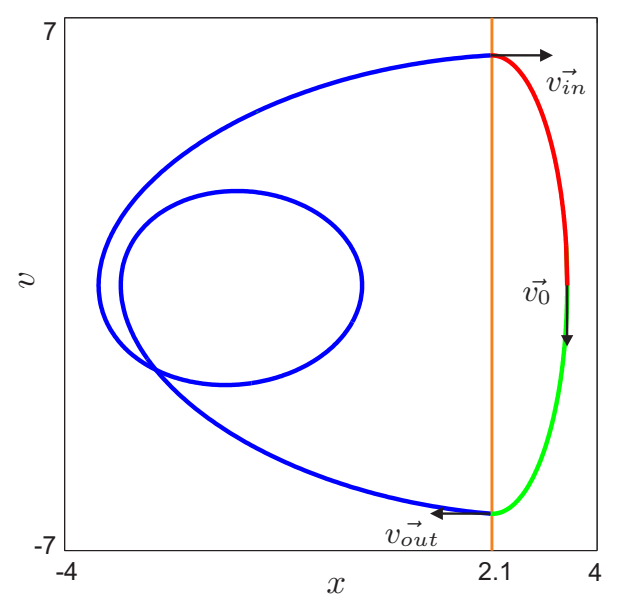

Figure 3.1: (colour online) Impact duration analysis. (a) Continuation of periodic motions of the impact oscillator [40]. The nondimensionalized parameters are the same as those detailed in Fig. 2.1 (a). Additionally, $k_{2}=66677.2 \mathrm{~N} / \mathrm{m}, m=1.0 \mathrm{~kg}$, and the continuation parameter, frequency ratio $\omega=0.35 \sim 0.95$. The solid and dashed curves denote stable and unstable solutions, respectively. Four subplots show the phase portraits of the four solid segments which are plotted with the corresponding colours. Bifurcation points (GR for grazing bifurcation, $\mathrm{F}$ for fold bifurcation, and PD for period-doubling bifurcation) are marked by X's. Panel (b) shows the phase portrait when $\omega=0.85$ in panel (a). This period-one one-impact trajectory is divided into three parts: no impact (blue), loading (red), and unloading(green). $\overrightarrow{v_{i n}}, \overrightarrow{v_{0}}, \overrightarrow{v_{\text {out }}}$ are the velocities at the start position of impact, zero velocity position, and the end position of impact, respectively.

where

$$
\begin{aligned}
& Q_{2}=\exp \left(-\xi\left(\tau-\tau_{n}\right)\right) \sqrt{A_{2}^{2}+B_{2}^{2}}, \\
& \phi_{2}=-\gamma_{2} \tau_{n}+\arctan \left(\frac{A_{2}}{B_{2}}\right) .
\end{aligned}
$$

From Eq. 3.2 to know, the impact motion is controlled by two frequencies: $\omega$ and $\gamma_{2}$. Their corresponding periods can be expressed as:

$$
T_{\omega}=\frac{2 \pi}{\omega}=\frac{\sqrt{\left(k_{1} / m\right)}}{f_{e}}, \quad T_{\gamma_{2}}=\frac{2 \pi}{\gamma_{2}}=\frac{2 \pi}{\sqrt{1+\beta-\xi^{2}}} .
$$

If the damping parameter $\xi^{2}$ is small enough to be neglected, and the stiffness of the impacted beam is much larger than the structure stiffness of the oscillator, i.e. $k_{2} \gg k_{1}$ or $\beta \gg 1$, the ratio between $T_{\omega}$ and $T_{\gamma_{2}}$ can be expressed as:

$$
\frac{T_{\omega}}{T_{\gamma_{2}}}=\frac{1 / f_{e}}{2 \pi / \sqrt{k_{2} / m}}=\frac{T_{e}}{T_{2}}
$$

where $T_{e}$ is the excitation period, and $T_{2}$ is the natural period of the oscillator-beam system. Namely, the impact duration is controlled by both $T_{e}$ and $T_{2}$.

Generally, comparing with $T_{e}$, the impact duration of a period-one one-impact motion is quite short, the influence of $T_{e}$ during the transient impact can be further neglected. Under such circumstances, the impact duration is only determined by $T_{2}$. As mentioned previously, from a geometric point of view, the impact phase experienced an approximate half of a harmonic period. Here, the harmonic period is the natural period of the oscillator-beam system, $T_{2}$. Since $T_{2}$ is determined by $m$ and $k_{2}$, the impact duration is constant if neither $m$ nor $k_{2}$ is changed, like the red segment shown in Fig. 3.1 (a). As a 
comparison, for the motion of the solid black segment shown in Fig. 3.1 (a), two impacts occur in each excitation period, the influence of the excitation period $T_{e}$ can not be neglected during the relatively long impact duration. That's why the black segment displays a slight upward tendency. Therefore, the stable period-one one-impact motion is the ideal response to build the correlation between stiffness and impact duration for impacting systems.

Based on the above analysis, and taking the position of zero velocity $\overrightarrow{v_{0}}$ shown in Fig. 3.1 (b) into consideration, the half $T_{2}$ can be further divided into a quarter of loading segment and a quarter of unloading segment. Therefore, the impact duration can be analytically predicted as:

$$
t=\frac{T_{l}}{4}+\frac{T_{u}}{4}=\frac{\pi / 2}{\sqrt{\frac{k_{l}}{m}}}+\frac{\pi / 2}{\sqrt{\frac{k_{u}}{m}}}=\frac{\pi}{\sqrt{\frac{k_{2}}{m}}},
$$

where $T_{l}$ and $T_{u}$ represent the loading period and the unloading period, respectively, and $m$ is the mass of the oscillator. For the case shown in Fig. 3.1 (b), the analytical prediction of its impact duration can be calculated as $t=\frac{\pi}{\sqrt{66677.2 / 1}}=0.0122 s$, which is the same as its continuation result shown in Fig. 3.1 (a). This, to some extent, demonstrates the correction of the analytical prediction.

\subsection{Stiffness estimation based on experimental studies}

The experimental rig described in [41] and shown in Fig. A.1 was used to obtain experimental data, to validate the analysis presented in Section. 3.1. Period-one one-impact motion was explored primarily for each experimental case by adjusting the excitation frequency. Subsequently, the period-one one-impact motion was investigated under different excitation frequencies, and the corresponding acceleration signals of the oscillator were recorded. In Fig. 3.2, the embedding parameters $(d=14, m=7)$ were obtained by nonlinear time series analysis. For the false nearest neighbour test, a $2 \%$ noise threshold was allowed. In Fig. 3.3, based on the tangent vector analysis of the reconstructed phase portrait, the average loading duration $(0.00667 \mathrm{~s})$, unloading duration $(0.00663 \mathrm{~s})$ were detected, and hence impact duration $(0.0130 \mathrm{~s})$ was calculated. Compared with the analytical prediction $(0.0122 \mathrm{~s})$, the experimental error is $6.56 \%$. As the same way, seven experimental cases were tested, and their average impact durations in period-one one-impact motions and the corresponding experimental errors are listed in Table. 3.1.

(a)

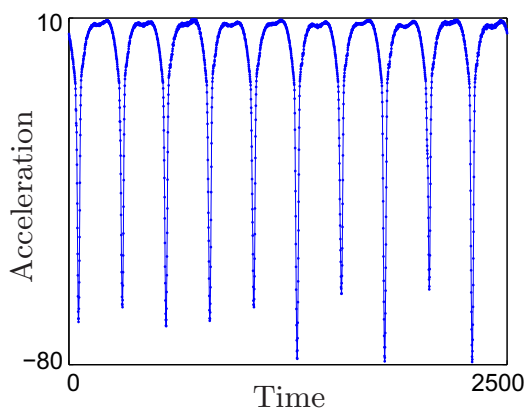

(b)

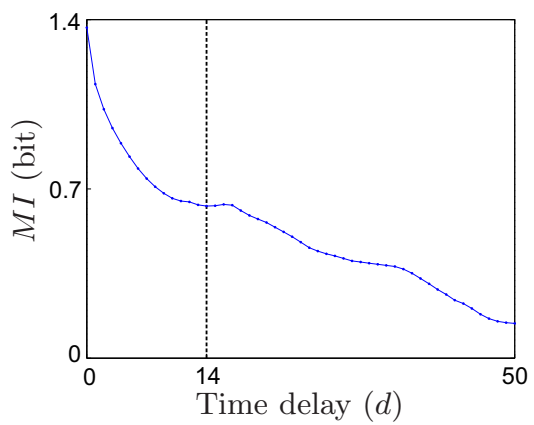

(c)

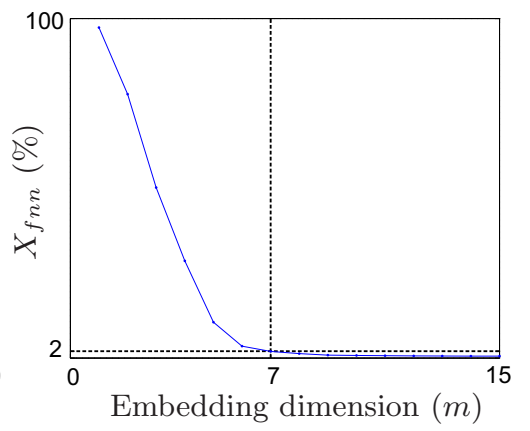

Figure 3.2: Nonlinear time series analysis of the 1st experimental case. (a) The experimental time series of the acceleration of the oscillator. Its experimental parameters correspond to the nondimensionalized parameters detailed in Fig. 2.1 (a). Subplots (b), (c) show the results of mutual information test $d=14$ and false nearest neighbour test $m=7$ with the noise threshold $\gamma=2 \%$. 
(a)

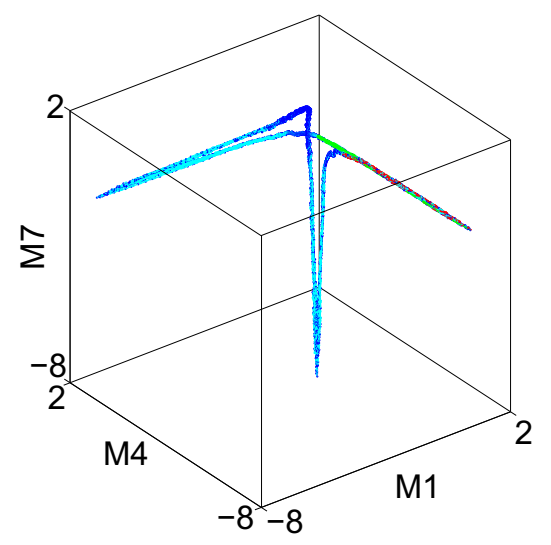

(c)

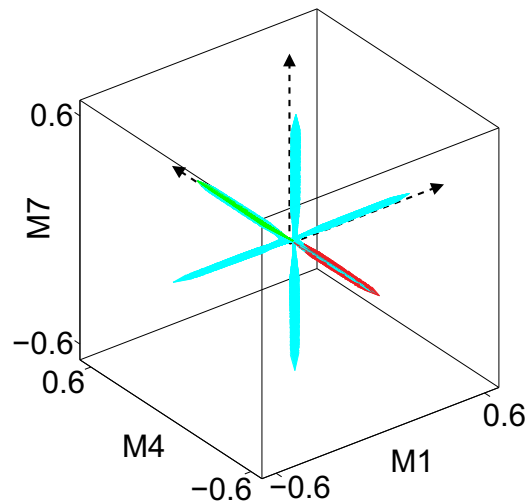

(b)
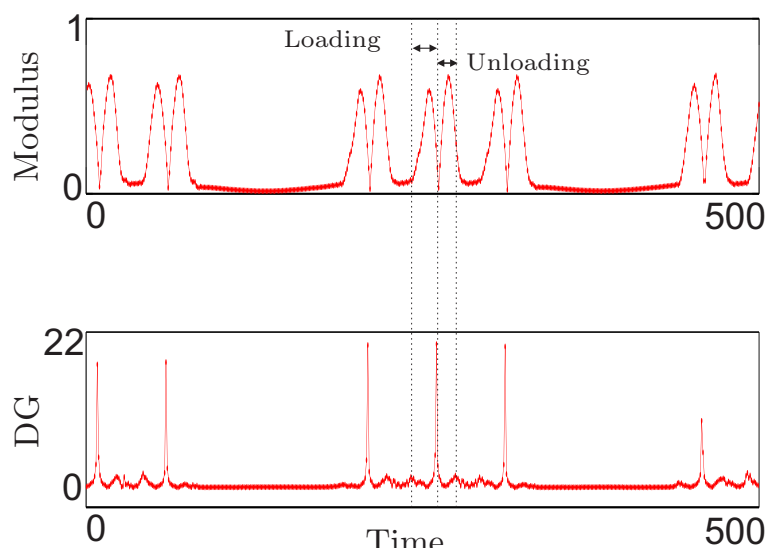

(d)
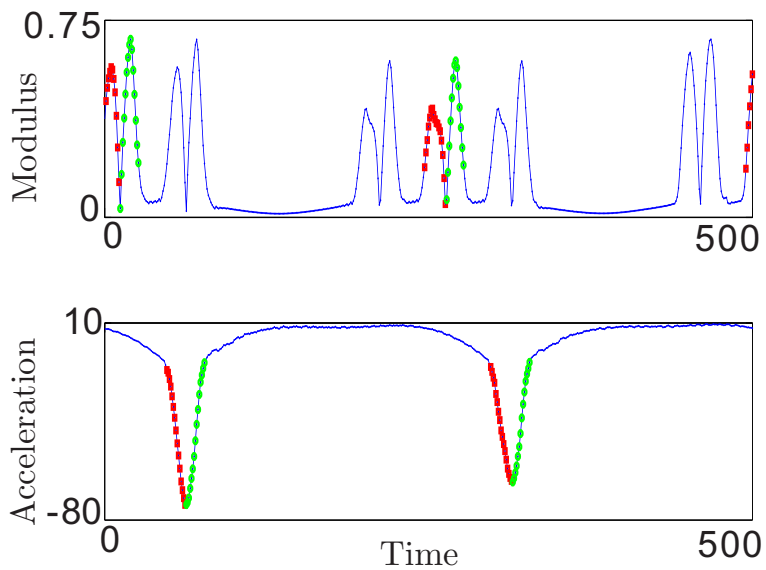

Figure 3.3: (colour online) Tangent vector analysis of the 1st experimental case. (a) The reconstructed 3D (1st, 4th, and 7th dimensions) phase portrait with $d=14$ and $m=7$. Specifically, the dark blue points constitute the 3D phase portrait, the light blue arrows show the tangent vectors. The marked red and green tangent vectors represent the loading and unloading phases in the 4th dimension. Subplot (b) shows the time series of modulus and the time series of direction gradient of tangent vectors. The tangent vectors depicted in (a) are rearranged at the origin in (c). Subplot (d) shows the result of the impact detection. The average loading duration is $0.00667 \mathrm{~s}$, and the average unloading duration is $0.00633 \mathrm{~s}$; hence, the average impact duration is calculated as 0.0130 s per excitation period.

Next, the obtained impact duration for each experimental case was used to estimate the corresponding stiffness. Before the stiffness estimation, the correlation curve between stiffness and impact duration is necessary. According to the analytical formula introduced in Eq. 3.6, a blue curve is depicted in Fig. 3.4. In order to verify the analytical curve, numerical simulation was performed, and the red curve shows its result. The blue and red curves overlap with each other, which demonstrates the accuracy of the analytical prediction. The experimental results, shown as green dots, display a similar trend as these two curves.

The stiffness estimation procedure is illustrated via the 1st and 7th experimental cases shown in Fig. 3.4. For the 1 st case, its measured stiffness is $k_{2}=66.68 \mathrm{kN} / \mathrm{m}$; while the estimated stiffness according to the detected impact duration $(t=0.0130 \mathrm{~s})$ is $k_{2}=61.65 \mathrm{kN} / \mathrm{m}$, hence the estimation error is $7.54 \%$. For the 7 th case, its measured stiffness is $k_{2}=170.99 \mathrm{kN} / \mathrm{m}$; while the estimated stiffness according to the detected impact duration $(t=0.0075 \mathrm{~s})$ is $k_{2}=175.19 \mathrm{kN} / \mathrm{m}$, hence its estimation error is $2.64 \%$. Other experimental cases were analysed with the same procedure. The estimation results and the corresponding 
Table 3.1: Result statistics and error analysis. For each experimental case, its analytical, simulated, and average experimental impact durations were obtained, and the corresponding experimental errors were calculated. According to the average experimental impact duration for each case, the stiffness of the impacted beam was estimated, and it was compared with its measured quasi-static stiffness.

\begin{tabular}{|c|ccc|cc|ccc|}
\hline \multirow{2}{*}{ Case } & \multicolumn{2}{|c|}{ Impact duration (s) } & \multicolumn{2}{c|}{ Error (\%) } & \multicolumn{3}{c|}{ Stiffness } \\
\cline { 2 - 10 } & analysis & simulation & exp. & $\begin{array}{c}\text { exp.- } \\
\text { analysis }\end{array}$ & $\begin{array}{c}\text { exp.- } \\
\text { simulation }\end{array}$ & $\begin{array}{c}\text { measured } \\
(\mathbf{k N} / \mathbf{m})\end{array}$ & $\begin{array}{c}\text { estimated } \\
(\mathbf{k N} / \mathbf{m})\end{array}$ & $\begin{array}{c}\text { error } \\
(\%)\end{array}$ \\
\hline 1 & 0.0122 & 0.0122 & 0.0130 & 6.56 & 6.56 & 66.68 & 61.65 & 7.54 \\
2 & 0.0115 & 0.0115 & 0.0121 & 5.22 & 5.22 & 74.65 & 68.45 & 8.30 \\
3 & 0.0108 & 0.0108 & 0.0117 & 8.33 & 8.33 & 84.04 & 73.69 & 12.32 \\
4 & 0.0102 & 0.0102 & 0.0105 & 2.94 & 2.94 & 95.23 & 89.64 & 5.87 \\
5 & 0.0093 & 0.0093 & 0.0096 & 3.23 & 3.23 & 113.87 & 107.65 & 5.46 \\
6 & 0.0085 & 0.0085 & 0.0090 & 5.88 & 5.88 & 137.23 & 123.75 & 9.83 \\
7 & 0.0076 & 0.0076 & 0.0075 & 1.32 & 1.32 & 170.99 & 175.19 & 2.46 \\
\hline Mean \\
Standard deviation
\end{tabular}

errors are listed in Table. 3.1. The average stiffness estimation error of this method is $7.39 \pm 3.20 \%$.

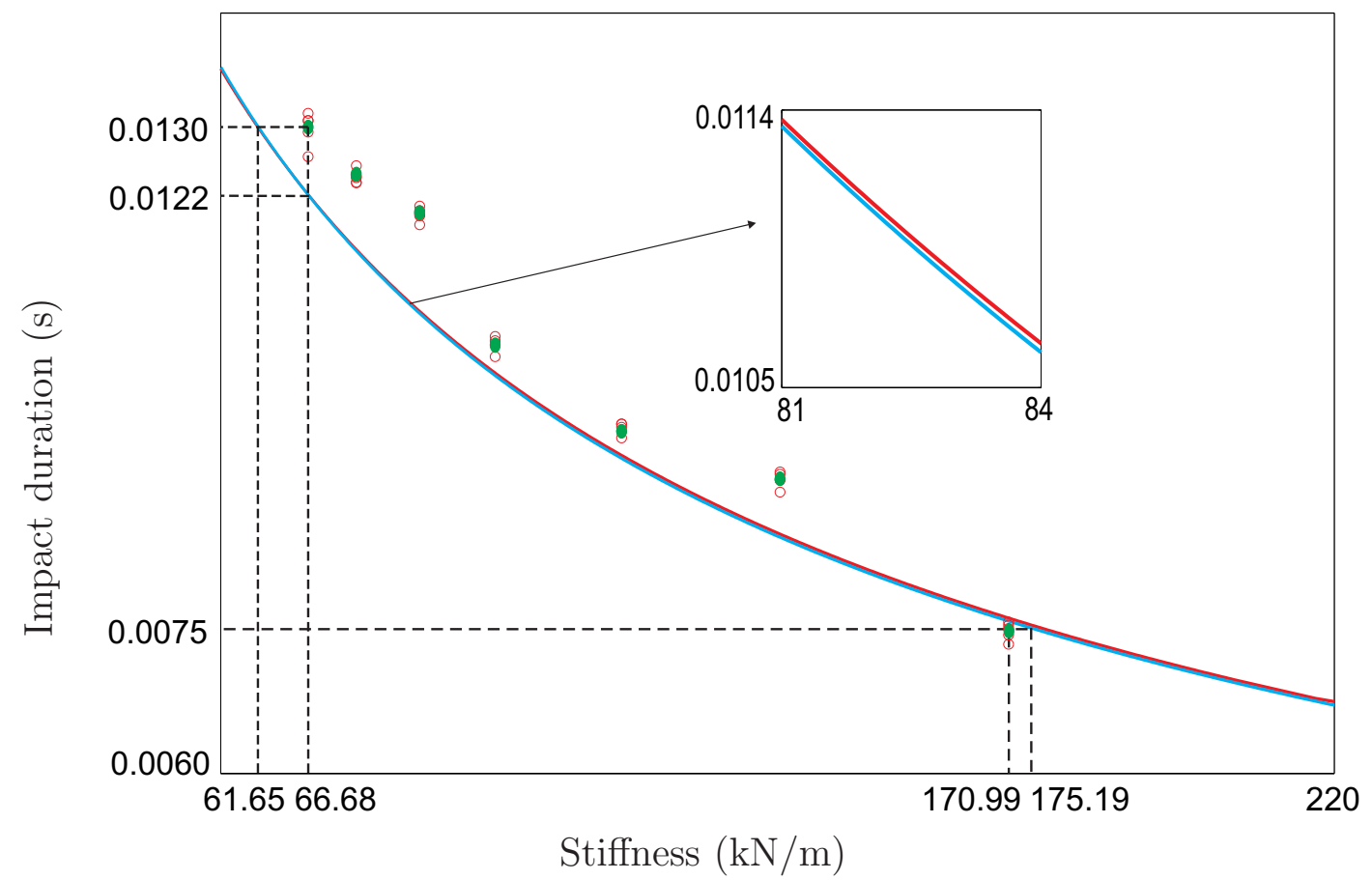

Figure 3.4: (colour online) Stiffness estimation. The blue curve is the analytical prediction, and the red curve is the corresponding numerical simulation. A blow-up in the top right corner shows the closeness between the blue and red curves. The red circles show the detected impact durations from experimental data. For each experimental case, the average impact duration is calculated, and is represented by a green dot. For the 1st experimental case whose measured stiffness $k_{2}=66.68 \mathrm{kN} / \mathrm{m}$, its analytical impact duration is $0.0122 \mathrm{~s}$. Based on the detected impact duration from experimental data, $t=0.0130 \mathrm{~s}$, the stiffness of the 1 st case is estimated as $k_{2}=61.65 \mathrm{kN} / \mathrm{m}$. For the 7 th case with the measured stiffness $k_{2}=170.99 \mathrm{kN} / \mathrm{m}$, the estimated stiffness is $k_{2}=175.19 \mathrm{kN} / \mathrm{m}$ according to the detected impact duration $t=0.0075 \mathrm{~s}$. 


\section{Stiffness identification of the rock impacting system}

The correlation between the stiffness of the impacted beam and its impact duration has been explored in Section 3. Meanwhile, the feasibility of applying this correlation to estimate the stiffness of the beam according to the detected impact duration has been demonstrated. In this section, rock is considered as the impacted object; its different stiffnesses in loading and unloading phases are described by a linear rock contact law, which is based on a series of quasi-static rock deformation tests. According to this rock contact law, a $2 \frac{1}{2}$-DOF rock impacting system is studied, to simulate the actual percussive drilling condition. The proposed dynamic method of stiffness identification in Section 3 will be further verified by using this new system.

\subsection{Rock contact law}

In order to simulate the deformation of the drilled rock, a series of quasi-static rock deformation tests were performed using INSTRON 1185. Three indenters with different contact angles and three rock samples with different in-situ elastic modulus were combined mutually to do these tests, see Fig. 4.1. For each rock-indenter combination, the loading and unloading operations were repeated ten times. The experimental data for the sandstone2-indenter1 combination are depicted in Fig. 4.2 (a). Among its ten tests, the rock sample was impacted in the first contact; then, it was compressed during the following five contacts; finally, both its loading and unloading stiffnesses reached relatively constant values in the last four cycles. For each test, the force-penetration correlations of the loading and unloading segments were fitted linearly; e.g., the linear fitting of the tenth test is displayed in Fig. 4.2 (b), the obtained rock deformation parameters are $k_{l}=21.55 \mathrm{kN} / \mathrm{mm}$ and $k_{u}=25.36 \mathrm{kN} / \mathrm{mm}$ with the linear geometric exponents $n_{l}=n_{u}=1$. Table 4.1 lists the ten fitting results of the sandstone2-indenter 1 combination, and the stable loading and unloading stiffnesses were obtained by calculating the average measurements of the last four tests. Using different rock-indenter combinations, nine experimental cases were tested, their average loading and unloading stiffnesses in the stable condition are listed in Table 4.2.

(a)

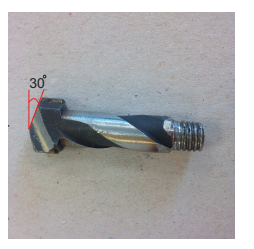

(b)

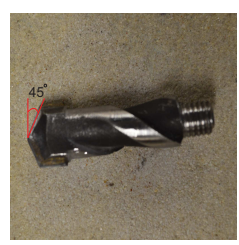

(c)

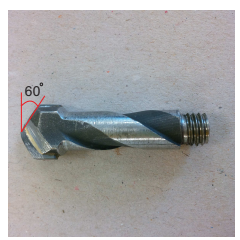

(d)

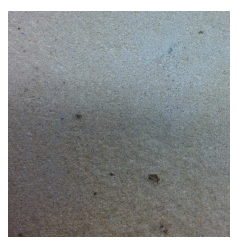

(e)

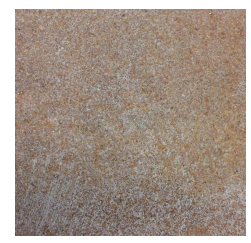

(f)

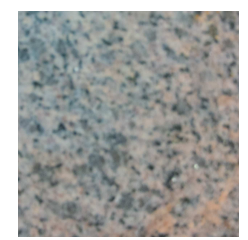

Figure 4.1: (colour online) Indenters and rock samples for quasi-static rock deformation tests. (a) Indenter1, (b) indenter2, (c) indenter3, (d) sandstone1, (e) sandstone2, and (f) granite. The contact angles of indenters and the in-situ elastic modulus of rock samples are listed in Table 4.2.

Table 4.1: Linear fitting results of the loading and unloading stiffnesses for the ten tests of the sandstone2-indenter1 combination. The repeated tests can be divided into three phases: rock impact, rock compression, and stable condition.

\begin{tabular}{|c|c|ccccc|ccccc|}
\hline Phase & impact & \multicolumn{4}{|c|}{ compression } & \multicolumn{5}{c|}{ stable } \\
\hline Test series & 1 st & 2nd & 3rd & 4th & 5th & 6th & 7 th & 8th & 9th & 10th & Average \\
\hline$k_{l}(\mathbf{k N} / \mathbf{m m})$ & 5.42 & 17.14 & 15.95 & 18.04 & 19.07 & 18.44 & 21.37 & 22.03 & 20.67 & 21.55 & $21.40 \pm 0.56$ \\
\hline$k_{u}(\mathbf{k N} / \mathbf{m m})$ & 21.57 & 21.75 & 23.55 & 22.37 & 25.65 & 23.95 & 24.24 & 24.25 & 26.35 & 25.36 & $25.05 \pm 1.01$ \\
\hline
\end{tabular}


(a)

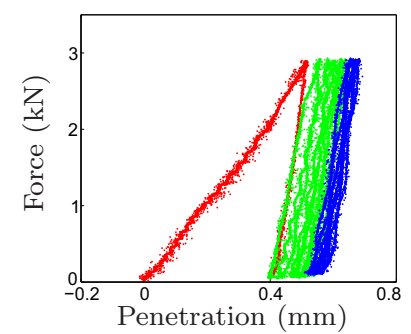

(b)

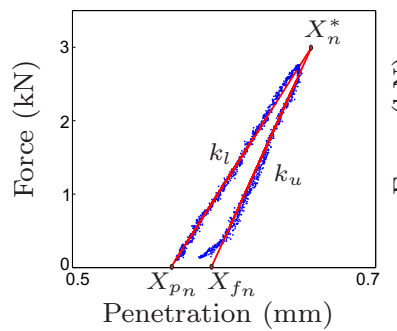

(c)

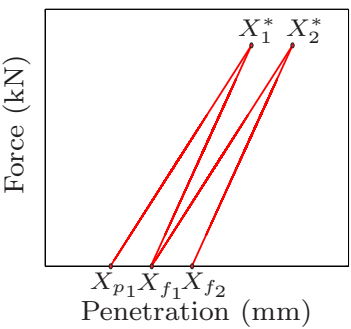

(d)

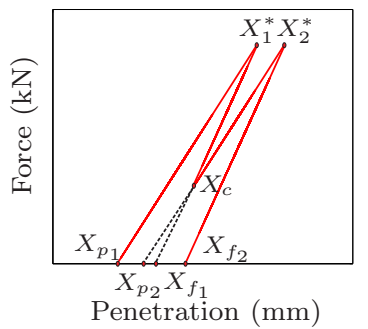

Figure 4.2: (colour online) Plots of force-penetration correlation of the sandstone2-indenter1 combination. (a) The experimental results of the ten tests. The red dots represent the first contact (impact), the green dots show the second to the sixth tests (compression), and the blue dots display the last four tests (stable). Subplot (b) shows the force-penetration correlation of the tenth test, including the experimental data (blue dots) and the linear fitting results (red lines). Based on the fitted force-penetration correlations, the rock contact model for the standard period-one one-impact motion is shown in subplot (c), and the model for the multiple-impact condition is shown in subplot (d).

Table 4.2: Experimental cases and their loading and unloading stiffnesses. Different experimental case is formed according to different rock-indenter combination. The loading stiffness $k_{l}$ and unloading stiffness $k_{u}$ are the averages of their corresponding stiffnesses in the stable condition.

\begin{tabular}{|ccccccc|}
\hline Case & \multirow{2}{*}{ Rock } & $\begin{array}{c}\text { Elastic } \\
\text { modulus }\end{array}$ & Indenter & $\begin{array}{c}\text { Contact } \\
\text { angle }\end{array}$ & $\begin{array}{c}\text { Load stiffness } \\
k_{l}(\mathrm{kN} / \mathrm{mm})\end{array}$ & $\begin{array}{c}\text { Unload stiffness } \\
k_{u}(\mathrm{kN} / \mathrm{mm})\end{array}$ \\
\hline 1 & \multirow{3}{*}{$4.80 \mathrm{GPa}$} & indenter1 & $30^{\circ}$ & $12.07 \pm 0.35$ & $13.70 \pm 0.37$ \\
2 & sandstone1 & indenter2 & $45^{\circ}$ & $11.82 \pm 0.25$ & $13.90 \pm 0.21$ \\
3 & & indenter3 & $60^{\circ}$ & $11.72 \pm 0.30$ & $13.41 \pm 0.38$ \\
\hline 4 & \multirow{2}{*}{ s.5. } & indenter1 & $30^{\circ}$ & $21.40 \pm 0.56$ & $25.05 \pm 1.01$ \\
5 & \multirow{3}{*}{ sandstone2 } & indenter2 & $45^{\circ}$ & $32.73 \pm 2.00$ & $40.64 \pm 2.01$ \\
6 & & indenter3 & $60^{\circ}$ & $22.03 \pm 0.47$ & $27.14 \pm 1.84$ \\
\hline 7 & \multirow{2}{*}{ granite } & \multirow{2}{*}{$52.16 \mathrm{GPa}$} & indenter1 & $30^{\circ}$ & $13.65 \pm 0.22$ & $15.15 \pm 0.32$ \\
8 & & indenter2 & $45^{\circ}$ & $11.46 \pm 0.18$ & $13.06 \pm 0.42$ \\
9 & & indenter3 & $60^{\circ}$ & $13.62 \pm 0.40$ & $14.91 \pm 0.33$ \\
\hline
\end{tabular}

The force-penetration correlation shown in Fig. 4.2 (b) can be written as [27]:

$$
k_{l}\left(X_{n}^{*}-X_{p_{n}}\right)^{n_{l}}=k_{u}\left(X_{n}^{*}-X_{f_{n}}\right)^{n_{u}} .
$$

For a one-impact motion, the final progression $X_{f_{n}}$ can be predicted at the end position of the current loading phase as:

$$
X_{f_{n}}=X_{n}^{*}-\left[\frac{k_{l}\left(X_{n}^{*}-X_{p_{n}}\right)^{n_{l}}}{k_{u}}\right]^{1 / n_{u}}
$$

where $\mathrm{n}=1,2$ in Fig. 4.2 (c). The obtained final progression in the current unloading phase $X_{f_{n}}$ is also the start position of the next loading phase $X_{p_{n+1}}$, e.g., $X_{p_{2}}=X_{f_{1}}$.

Another possible condition is the multiple-impact motion, the corresponding force-penetration correlation is shown in Fig. 4.2 (d). Specifically, during the unloading process, before the oscillator reaching the predicted final progression $X_{f_{n}}$, its velocity changes sign. Under such circumstances, the start position of the next loading phase $X_{p_{n+1}}$ is different from $X_{f_{n}}$; hence a virtual $X_{p_{n+1}}$ needs to be recalculated according to the position of the direction changing point $X_{c}$ as:

$$
X_{p_{n+1}}=X_{c}-\left[\frac{k_{u}\left(X_{c}-X_{f_{n}}\right)^{n_{u}}}{k_{l}}\right]^{1 / n_{l}}
$$


where $\mathrm{n}=1$ in Fig. $4.2(\mathrm{~d})$.

\subsection{Physical and mathematical models}

The experimental setup of the $2 \frac{1}{2}$-DOF rock impacting system was built by Sayah [42] in University of Aberdeen. The details of the experimental setup are shown in Fig. 4.3, based on which, the physical model of the new system is developed and shown in Fig. 4.4. Specifically, the drillbit assembly is modeled by the mass $m_{2}$, and the mass $m_{1}$ accounts for the equipment above the drillbit assembly. The slider represents the rock which obeys the rock contact law proposed in subsection 4.1. Compare with the experimental setup, the physical model does not take the rotation of rock into consideration; by this way, the physical model focuses on the axial interaction between the drillbit assembly and the rock sample.

(a)

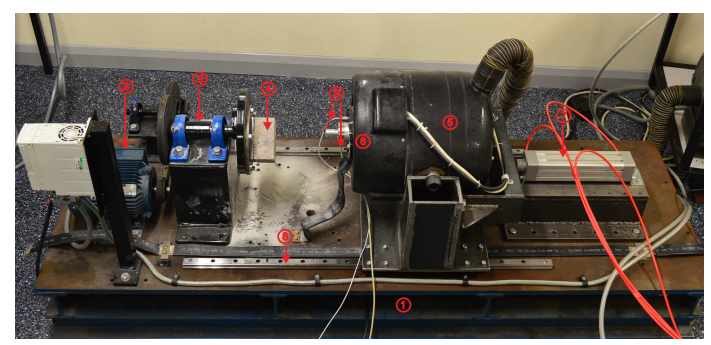

(b)

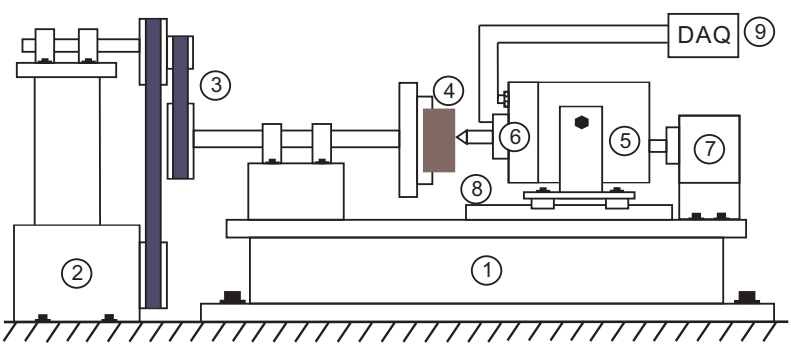

Figure 4.3: (colour online) Experimental setup [43]. (a) Photo of the experimental setup, (b) schematic of the experimental setup. (1) is a base table. (2) is a DC electrical motor which can rotate rock samples (4) through a group of pulleys (3). (5) is an electro-dynamical shaker that vibrates the drillbit assembly (6) horizontally. (7) is a pneumatic actuator designed for providing static force. The shaker can move along a guide (8) to make the drillbit assembly contact with rock samples. The acceleration and impact force of the drillbit assembly are measured by an accelerometer and a load cell, respectively; then the measured signals are collected by a data acquisition system (9).

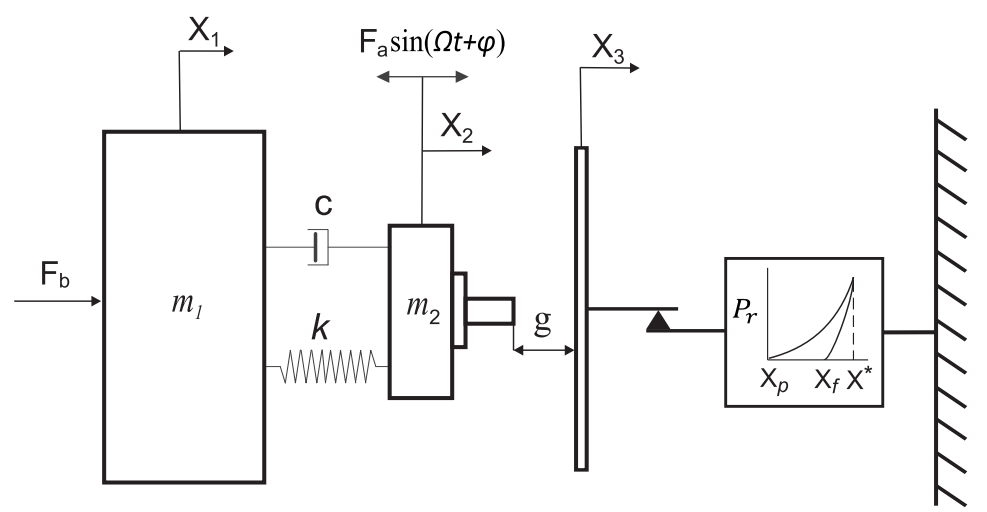

Figure 4.4: Physical model of the $2 \frac{1}{2}$-DOF rock impacting system. A static force $F_{b}$ is acted on mass $m_{1}$; mass $m_{2}$ is subjected to a harmonic excitation force $F_{a}$ with angular velocity $\Omega$ and phase shift $\varphi$. The two masses $m_{1}$ and $m_{2}$ are connected by a structure spring $k$ and a damper $c$. The half DOF refers to the massless slider (representing rock surface), since it is governed by a first order equation only. $g$ is the initial gap between $m_{2}$ and a slider. The displacements of $m_{1}$, $m_{2}$, and the slider are denoted by $X_{1}, X_{2}$, and $X_{3}$, respectively. For the rock contact part, $P_{r}$ is the stress from rock deformation. $X_{p}, X_{f}$, and $X^{*}$ are the positions of the slider at the start, the end and the maximal penetration in one impact period.

According to the rock contact law developed in subsection 4.1, the initial position of the loading phase $X_{p}$ and the final progression in the unloading phase $X_{f}$ vary in an impulsive manner. However, in order 
to integrate them into ODEs, rather than the impulsive variations, their differential equations need to be developed to describe their variations continuously. According to the drillbit-rock interaction, this system operates under any of the three modes: no contact, loading, or unloading. In no contact phase, both $X_{p}$ and $X_{f}$ remain invariable; hence, $\dot{X}_{p}=0$ and $\dot{X}_{f}=0$. During the loading phase, $X_{p}$ still remains invariable, i.e. $\dot{X}_{p}=0$; but $X_{f}$ needs to be calculated constantly until $X_{2}$ reaches the maximal progression. Refer to Eq. 4.1, the differential equation of $X_{f}$ can be expressed as:

$$
\dot{X}_{f}=\left(1-\frac{k_{l} n_{l}\left(X_{2}-X_{p}\right)^{n_{l}-1}}{k_{u} n_{u}\left(X_{2}-X_{f}\right)^{n_{u}-1}}\right) \dot{X}_{2} .
$$

For the unloading phase, $X_{f}$ remains constant, i.e. $\dot{X}_{f}=0$; but the new $X_{p}$ for the next impact period needs to be calculated, still refer to Eq. 4.1, the differential equation of $X_{p}$ can be expressed as:

$$
\dot{X}_{p}=\left(1-\frac{k_{u} n_{u}\left(X_{2}-X_{f}\right)^{n_{u}-1}}{k_{l} n_{l}\left(X_{2}-X_{p}\right)^{n_{l}-1}}\right) \dot{X}_{2} .
$$

Based on the above analyses, the nondimensionalized equations of motion for this system can be written in a compact form:

$$
\left\{\begin{array}{l}
x_{1}^{\prime}=v_{1} \\
v_{1}^{\prime}=\alpha b-\alpha \beta\left(x_{1}-x_{2}\right)-2 \alpha \xi\left(v_{1}-v_{2}\right) \\
x_{2}^{\prime}=v_{2}, \\
v_{2}^{\prime}=a \sin (s+\varphi)-\beta\left(x_{2}-x_{1}\right)-2 \xi\left(v_{2}-v_{1}\right)-P_{1} P_{2}\left(x_{2}-x_{p}\right)^{n_{l}}-P_{1} P_{3}\left(1-P_{2}\right) \kappa\left(x_{2}-x_{f}\right)^{n_{u}}, \\
x_{3}^{\prime}=\left(P_{1} P_{2}+P_{1} P_{3}\left(1-P_{2}\right)\right) v_{2}, \\
x_{p}^{\prime}=P_{1}\left(1-P_{2}\right) P_{3}\left(1-\frac{\kappa n_{u}\left(x_{2}-x_{f}\right)^{n_{u}-1}}{n_{l}\left(x_{2}-x_{p}\right)^{n_{l}-1}}\right) v_{2}, \\
x_{f}^{\prime}=P_{1} P_{2}\left(1-\frac{n_{l}\left(x_{2}-x_{p}\right)^{n_{l}-1}}{\kappa n_{u}\left(x_{2}-x_{f}\right)^{n_{u}-1}}\right) v_{2}, \\
s^{\prime}=\omega
\end{array}\right.
$$

where $P_{1}, P_{2}, P_{3}$ stand for Heaviside step functions defined as:

$$
P_{1}=H\left(x_{2}-x_{p}\right), \quad P_{2}=H\left(v_{2}\right), \quad P_{3}=H\left(x_{2}-x_{f}\right),
$$

and $x_{1}^{\prime}, v_{1}^{\prime}, x_{2}^{\prime}, v_{2}^{\prime}, x_{3}^{\prime}, x_{p}^{\prime}, x_{f}^{\prime}, s^{\prime}$ denote differentiation with respect to the nondimensionalized time $\tau$. The nondimensionalized variables and parameters of this system are listed as follows:

$$
\begin{aligned}
& x_{1}=\frac{X_{1}}{\mu}, \quad x_{2}=\frac{X_{2}}{\mu}, \quad x_{3}=\frac{X_{3}}{\mu}, \quad x_{p}=\frac{X_{p}}{\mu}, \quad x_{f}=\frac{X_{f}}{\mu}, \\
& \tau=\Omega_{0} t, \quad \alpha=\frac{m_{2}}{m_{1}}, \quad \omega=\frac{\Omega}{\Omega_{0}}, \quad a=\frac{F_{a}}{m_{2} \mu \Omega_{0}^{2}}, \quad b=\frac{F_{b}}{m_{2} \mu \Omega_{0}^{2}}, \\
& \beta=\frac{k}{k_{l}} \mu^{1-n_{l}}, \quad \xi=\frac{c}{2 m_{2} \Omega_{0}}, \quad G=\frac{g}{\mu}, \quad \kappa=\left(\frac{k_{u}}{k_{l}}\right) \mu^{n_{u}-n_{l}}, \quad \Omega_{0}^{2}=\frac{k_{l}}{m_{2}} \mu^{n_{l}-1},
\end{aligned}
$$

where $\mu$ is a reference distance, and $\Omega_{0}$ is the natural frequency. $k_{l}, k_{u}$ are the stiffnesses of rock in the loading and unloading phases, $n_{l}$ and $n_{u}$ are their corresponding geometric exponents.

According to the established mathematical models, numerical integration (see Fig. 4.5 (a)) and numerical continuation (see Fig. 4.5 (b)) for the sandstone2-indenter1 combination were carried out. From 
both figures, a broad range $(58.82 \mathrm{~Hz} \sim 129.90 \mathrm{~Hz})$ of the stable period-one one-impact motion is observed. This segment is truncated by a torus bifurcation (TR2) on the left side and a period-doubling bifurcation (PD2) on the right side. Furthermore, this segment marked as black in Fig. 4.5 (b) remains horizontal, namely, this period-one one-impact response has a constant impact duration.

\subsection{Analytical prediction of impact duration}

For the analytical prediction of the impact duration for the $2 \frac{1}{2}$-DOF rock impacting system, the basic assumption is the same as that of the 1-DOF impact oscillator. Specifically, the impact duration of the period-one one-impact motion is constant, and it is determined by the natural period of the bit-rock system. Meanwhile, considering that the stiffnesses of rock are different in loading and unloading phases, the loading duration can be approximated as a quarter of the loading period; while the unloading duration is approximated as a quarter of the unloading period. Hence, the analytical predictions of the loading duration $\left(t_{l}\right)$ and unloading duration $\left(t_{u}\right)$ can be formulated as Eq. 3.6 as:

$$
\left\{\begin{array}{l}
t_{l}=\frac{T_{l}}{4}=\frac{\pi / 2}{\sqrt{\frac{k_{l}}{m_{2}}}} \\
t_{u}=\frac{T_{u}}{4}=\frac{\pi / 2}{\sqrt{\frac{k_{u}}{m_{2}}}}
\end{array}\right.
$$

Hence, the loading stiffness $\left(k_{l}\right)$ and the unloading stiffness $\left(k_{u}\right)$ can be estimated as:

$$
\left\{\begin{array}{l}
k_{l}=m_{2}\left(\frac{\pi / 2}{t_{l}}\right)^{2} \\
k_{u}=m_{2}\left(\frac{\pi / 2}{t_{u}}\right)^{2}
\end{array}\right.
$$

In order to verify the analytical prediction, the loading and unloading durations between the analytical prediction and the numerical simulation are depicted in Fig. 4.6. Neither the simulated loading duration nor the simulated unloading duration keeps absolutely constant in its period-one one-impact motions, and slight fluctuations can be observed when sweeping the excitation frequency from $80 \mathrm{~Hz}$ to $105 \mathrm{~Hz}$. The average prediction errors are $2.57 \pm 0.49 \%$ for the loading duration, and $1.26 \pm 0.55 \%$ for the unloading duration. The errors stay in an acceptable level, hence the feasibility of the analytical prediction is still secured. This verification is the foundation for the following rock stiffness estimation.

\subsection{Impact duration estimation}

As listed in Table 4.2, nine experimental cases were tested dynamically by using the experimental rig. For each case, the pneumatic actuator was set as 1 bar, the input excitation amplitude was remained constant as $450 \mathrm{mV}$, and the excitation frequency was varied from $80 \mathrm{~Hz}$ to $100 \mathrm{~Hz}$ with a $5 \mathrm{~Hz}$ interval. The acceleration signal about the sandstone2-indenter 1 case collected in $80 \mathrm{~Hz}$ was analysed by nonlinear time series analysis in Fig. 4.7 and by tangent vector analysis in Fig. 4.8. The obtained loading duration is $5.581 \times 10^{-4} \mathrm{~s}$, and the unloading duration is $5.257 \times 10^{-4} \mathrm{~s}$.

As a comparison, the acceleration obtained by numerical simulation with the same parameters was also analysed. The nonlinear time series analysis is shown in Fig. 4.9, and its tangent vector analysis is shown in Fig. 4.10. The obtained loading duration is $5.713 \times 10^{-4} \mathrm{~s}$, and the unloading duration is $5.375 \times 10^{-4} \mathrm{~s}$. 
(a)

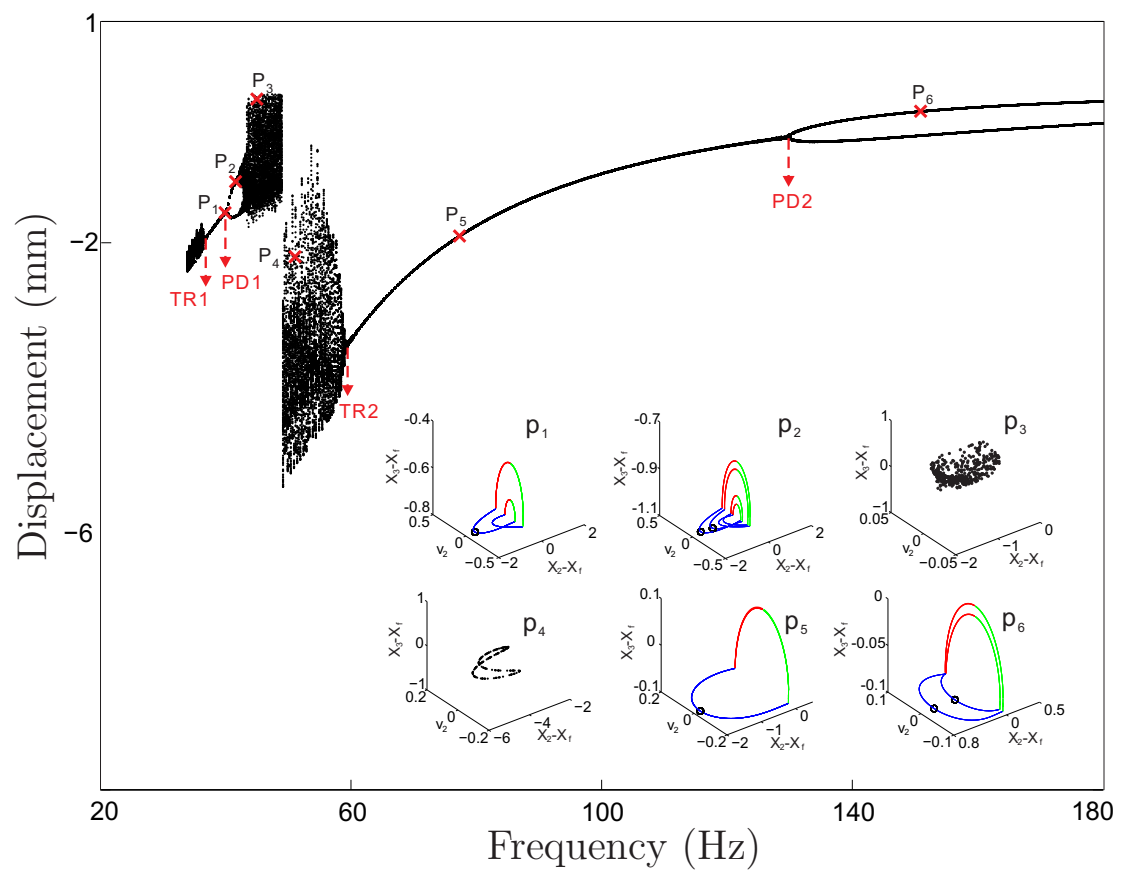

(b)

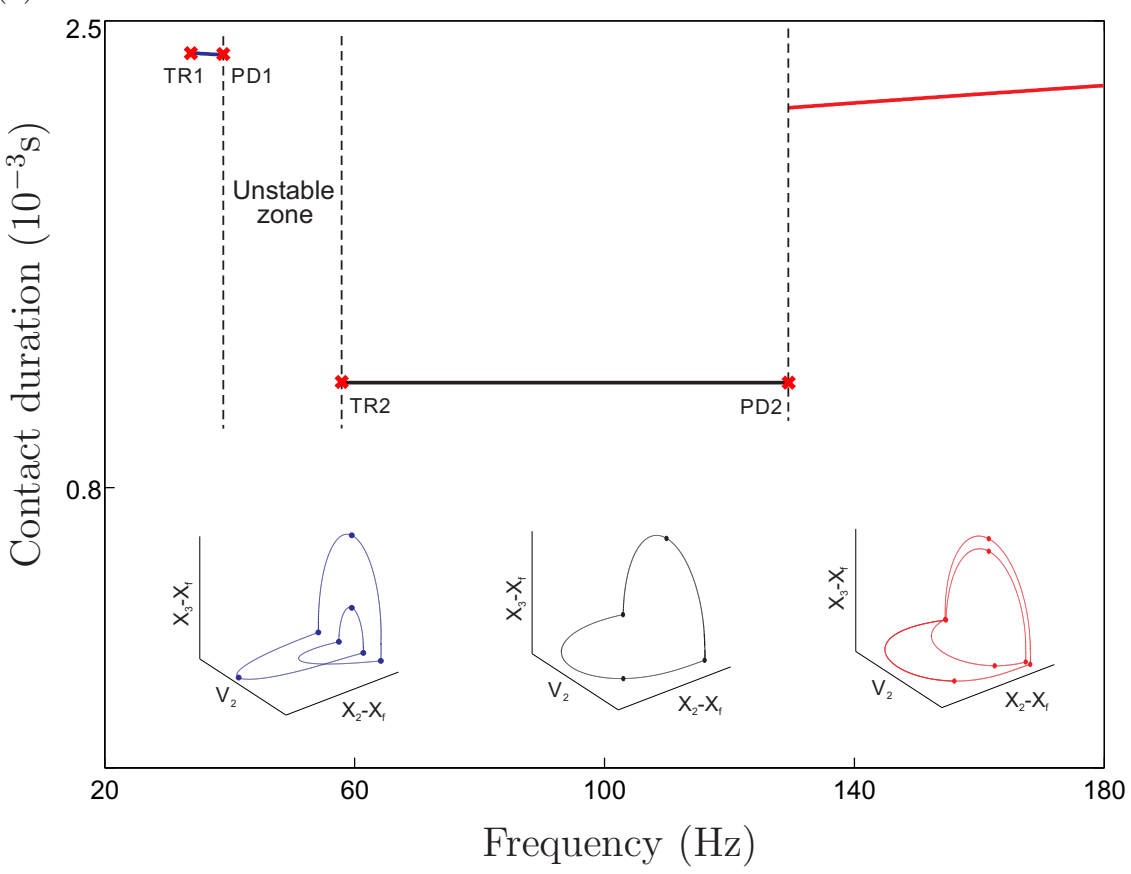

Figure 4.5: (colour online) Bifurcation analysis of the $2 \frac{1}{2}$-DOF rock impacting system with the excitation frequency range 35 180 Hz and the nondimensionalized parameters: $\alpha=0.0113, a=0.0133, b=0.0095, \beta=0.0033, \xi=0.0172, G=0.02$, $\kappa=1.1905$, and $\Omega_{0}=2645.8$. (a) Bifurcation diagram based on the numerical integration. Additional panels $\left(p_{1}-p_{6}\right)$ in the right-bottom corner show typical trajectories obtained at frequencies $40 \mathrm{~Hz}$ (period-one two-impact motion), $42 \mathrm{~Hz}$ (period-two four-impact motion), $44 \mathrm{~Hz}$ (stroboscopic map for chaos), $46 \mathrm{~Hz}$ (stroboscopic map for quasi-periodic motion), $80 \mathrm{~Hz}$ (period-one one-impact motion), $150 \mathrm{~Hz}$ (period-two two-impact motion), respectively. (b) Continuation of three stable periodic motions using TC-HAT. Three subplots show the 3D phase portraits belonging to the three segments which are depicted with the corresponding colours. They are obtained at frequencies $40 \mathrm{~Hz}, 80 \mathrm{~Hz}, 150 \mathrm{~Hz}$, respectively. The unstable zone corresponds to the period-doubling cascade to chaos and quasi-periodic motions, which can not be analysed by TC-HAT. In both subplots, bifurcation points (torus bifurcations TR1 at $36.60 \mathrm{~Hz}$, TR2 at $58.82 \mathrm{~Hz}$, and period-doubling bifurcations PD1 at $40.38 \mathrm{~Hz}, \mathrm{PD} 2$ at $129.90 \mathrm{~Hz}$ ) are marked. 
(a)

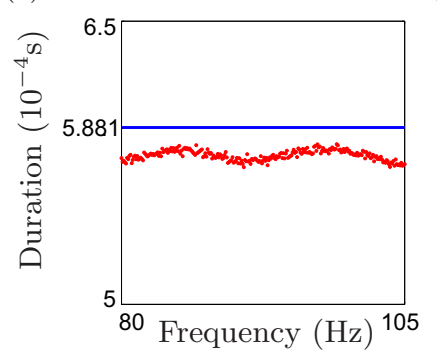

(b)

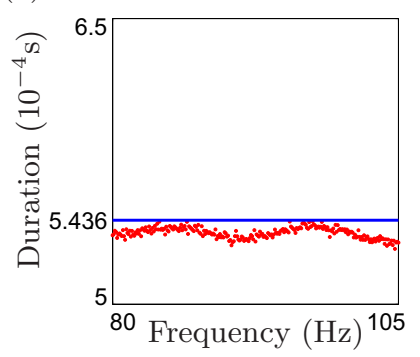

(c)

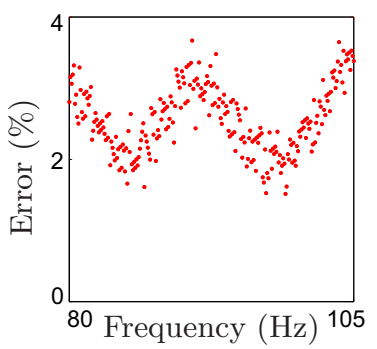

(d)

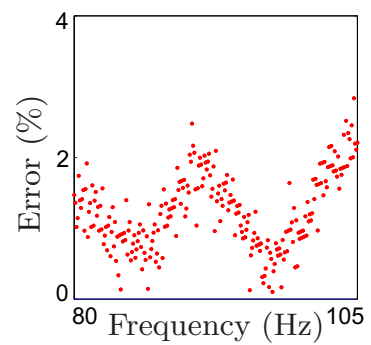

Figure 4.6: (colour online) Comparison of the analytical prediction and the numerical simulation for the loading and unloading durations. The same parameters as those detailed in Fig. 4.5 were used except the excitation frequency increases from $80 \mathrm{~Hz}$ to $105 \mathrm{~Hz}$. Subplot (a) shows the comparison of the loading durations. The blue line stands for the analytical prediction, and the red dots represent the simulated loading durations. Subplot (b) shows the comparison of the unloading durations. Subplot (c) displays the prediction errors of the loading durations, and the average error is $2.57 \pm 0.49 \%$. Subplot (d) displays the prediction errors of the unloading durations, and the average error is $1.26 \pm 0.55 \%$.

Comparing the detected experimental durations shown in Fig. 4.8 with the corresponding analytical predictions shown in Fig. 4.6, the experimental errors are 5.10\% for the loading duration, and $3.29 \%$ for the unloading duration. When the detected experimental durations are compared with the durations explored from the simulated acceleration (see Fig. 4.10), the experimental errors are $2.31 \%$ for the loading duration, and $2.20 \%$ for the unloading duration. Table 4.3 lists the durations of the analytical predictions, numerical simulations, and experiments for the nine study cases. The average experiment-analysis errors are $2.48 \pm 2.87 \%$ for the loading duration and $3.91 \pm 2.39 \%$ for the unloading duration. The average experiment-simulation errors are $3.43 \pm 0.74 \%$ for the loading duration, and $3.07 \pm 3.16 \%$ for the unloading duration.

Table 4.3: Analyses of the loading and unloading durations. The detected experimental loading and unloading durations are compared with the durations obtained by analytical predictions and numerical simulations. The corresponding experimentanalysis and experiment-simulation errors are calculated.

\begin{tabular}{|c|c|c|c|c|c|c|c|c|c|c|}
\hline \multirow{3}{*}{ Case } & \multicolumn{6}{|c|}{ Average duration $\left(10^{-4} \mathbf{s}\right)$} & \multicolumn{4}{|c|}{ Error (\%) } \\
\hline & \multicolumn{2}{|c|}{ analysis } & \multicolumn{2}{|c|}{ simulation } & \multicolumn{2}{|c|}{ experiment } & \multicolumn{2}{|c|}{$\begin{array}{l}\text { experiment } \\
\text {-analysis }\end{array}$} & \multicolumn{2}{|c|}{$\begin{array}{l}\text { experiment } \\
\text {-simulation }\end{array}$} \\
\hline & load & unload & load & unload & load & unload & load & unload & load & unload \\
\hline 1 & 7.831 & 7.351 & 7.513 & 7.225 & 7.859 & 8.021 & 0.36 & 9.11 & 4.60 & 11.02 \\
\hline 2 & 7.914 & 7.297 & 7.650 & 7.188 & 7.942 & 7.047 & 0.35 & 3.43 & 3.82 & 1.96 \\
\hline 3 & 7.947 & 7.430 & 7.675 & 7.274 & 8.003 & 7.089 & 0.70 & 3.55 & 3.18 & 0.64 \\
\hline 4 & 5.881 & 5.436 & 5.713 & 5.375 & 5.581 & 5.257 & 5.10 & 3.29 & 2.31 & 2.20 \\
\hline 5 & 4.756 & 4.268 & 4.636 & 4.278 & 4.769 & 4.107 & 0.27 & 3.77 & 2.87 & 4.00 \\
\hline 6 & 5.797 & 5.222 & 5.663 & 5.150 & 5.450 & 5.124 & 5.99 & 1.88 & 3.76 & 0.50 \\
\hline 7 & 7.364 & 6.990 & 7.100 & 6.809 & 7.334 & 6.634 & 0.41 & 5.09 & 3.30 & 2.57 \\
\hline 8 & 8.037 & 7.529 & 7.682 & 7.309 & 7.438 & 7.262 & 7.45 & 3.55 & 3.18 & 0.64 \\
\hline 9 & 7.372 & 7.046 & 7.055 & 6.864 & 7.251 & 7.014 & 1.64 & 0.45 & 2.78 & 2.19 \\
\hline \multicolumn{7}{|c|}{ Mean error } & 2.48 & 3.91 & 3.43 & 3.07 \\
\hline Standa & devia & & & & & & \pm 2.87 & \pm 2.39 & \pm 0.74 & \pm 3.16 \\
\hline
\end{tabular}


(a)

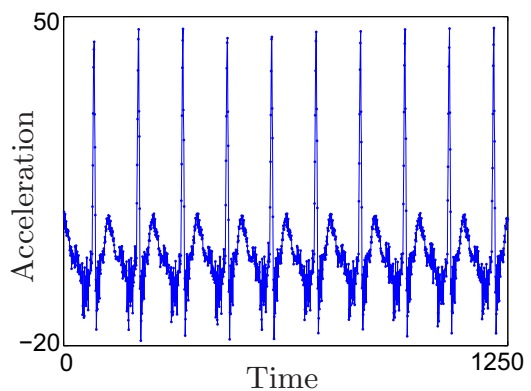

(b)

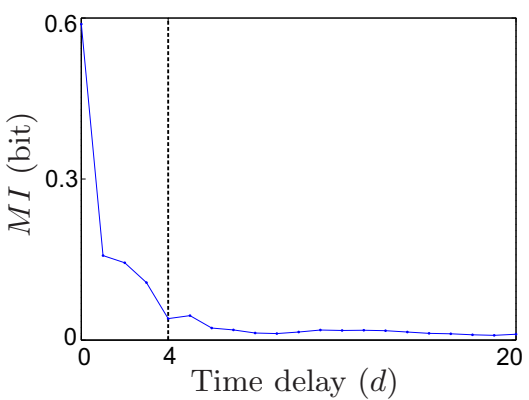

(c)

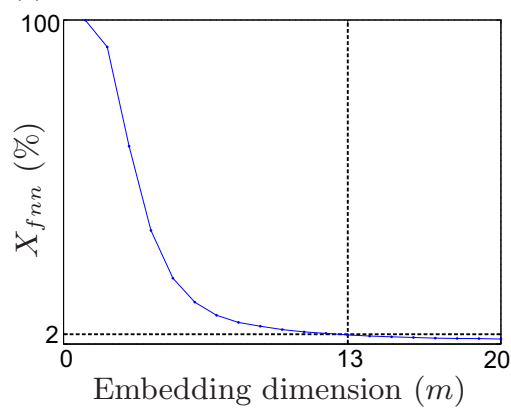

Figure 4.7: Nonlinear time series analysis of the experimental data. Subplot (a) is an acceleration time series collected in excitation frequency $80 \mathrm{~Hz}$. The dimensional parameters corresponding to the physical model shown in Fig. 4.4 are $F_{a}=279 \mathrm{~N}, F_{b}=200 \mathrm{~N}, c=272.68 \mathrm{~N} \cdot \mathrm{s} / \mathrm{m}, g=0.02 \mathrm{~mm}, m_{1}=265 \mathrm{~kg}, m_{2}=3 \mathrm{~kg}, k_{l}=21.40 \mathrm{kN} / \mathrm{mm}, k_{u}=25.05 \mathrm{kN} / \mathrm{mm}$, $k=0.07 \mathrm{kN} / \mathrm{mm}$. Subplots (b), (c) show the results of its mutual information test $d=4$ and its false nearest neighbour test $m=13$ with the noise threshold $\gamma=2 \%$.

(a)

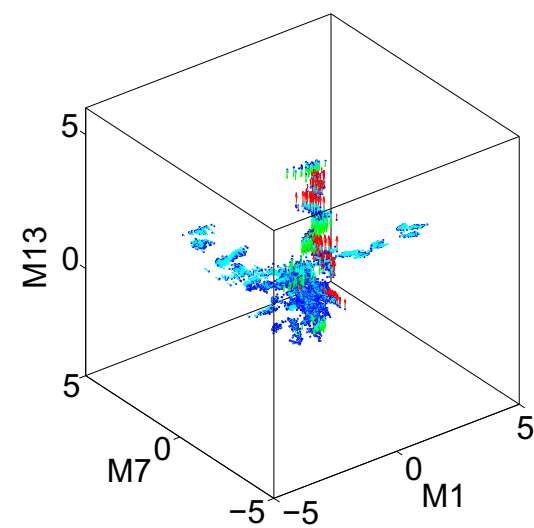

(c)

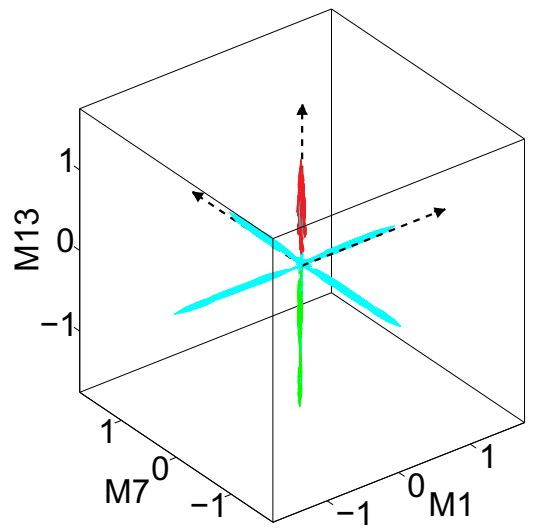

(b)
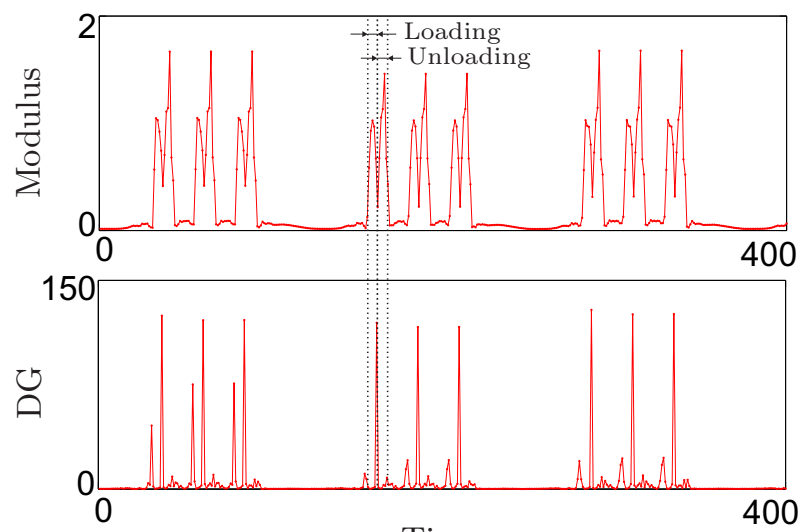

Time

(d)
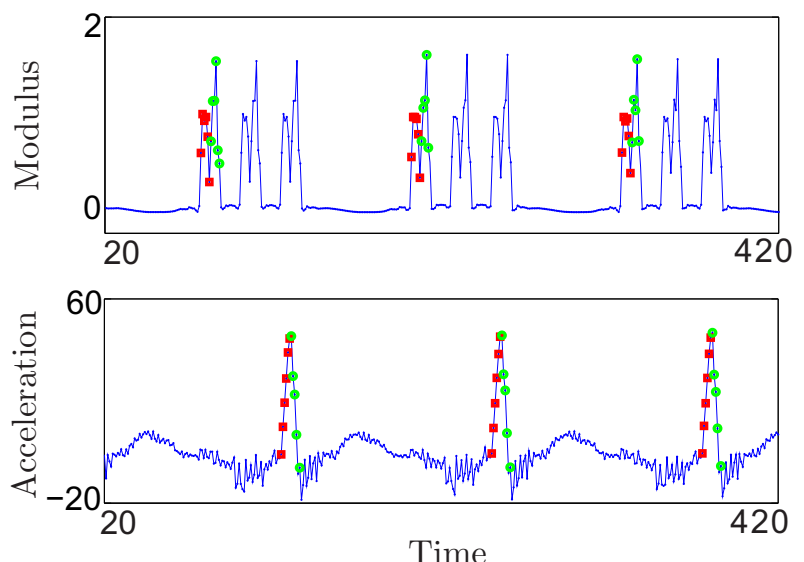

Figure 4.8: (colour online) Tangent vector analysis of the experimental data. (a) The reconstructed 3D (1st, 7th, and 13th dimensions) phase portrait with $d=4$ and $m=13$. Specifically, the dark blue points constitute the 3D phase portrait, the light blue arrows show the tangent vectors. The marked red and green tangent vectors represent the loading and unloading phases in the 13th dimension. Subplot (b) shows the time series of modulus, and the time series of direction gradient of tangent vectors. The tangent vectors depicted in subplot (a) are rearranged at the origin in subplot (c). Subplot (d) shows the result of the impact detection. The average loading duration is $5.581 \times 10^{-4} \mathrm{~s}$ per excitation period, and the average unloading duration is $5.257 \times 10^{-4}$ s per excitation period. 
(a)

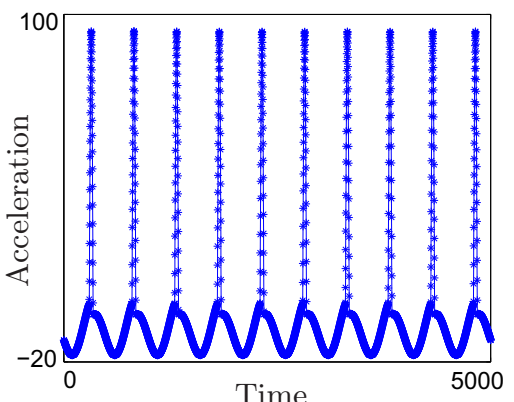

(b)

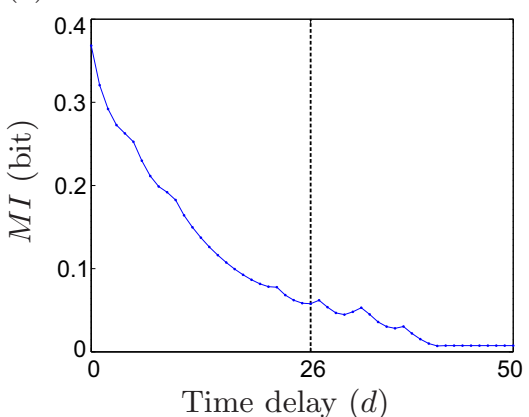

(c)

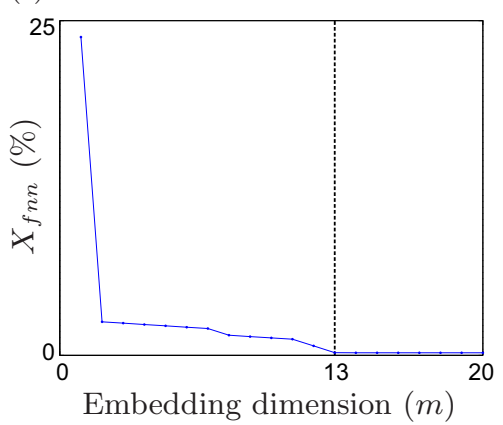

Figure 4.9: Nonlinear time series analysis of the simulated data. Subplot (a) is the time series of acceleration simulated with the equal nondimensionalized parameters as those detailed in Fig. 4.7 (a). Subplots (b), (c) show the results of its mutual information test $d=26$ and its false nearest neighbour test $m=13$, respectively.

(a)

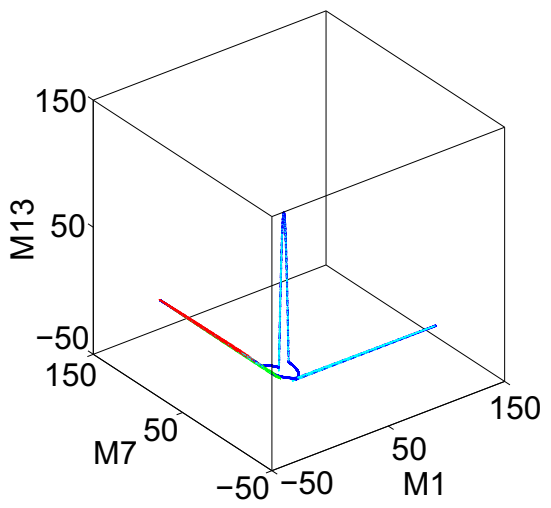

(c)

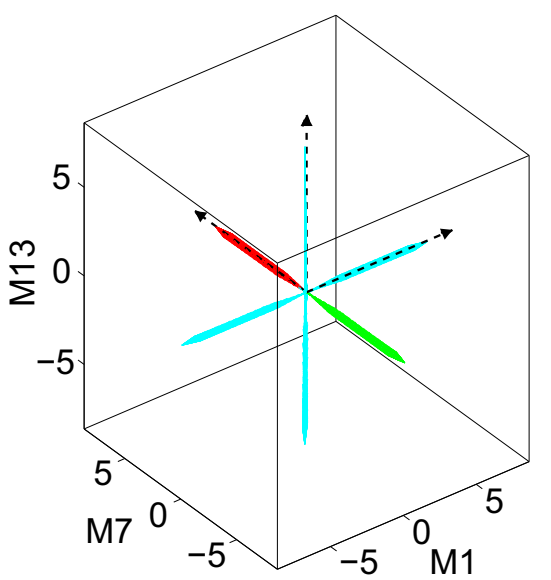

(b)

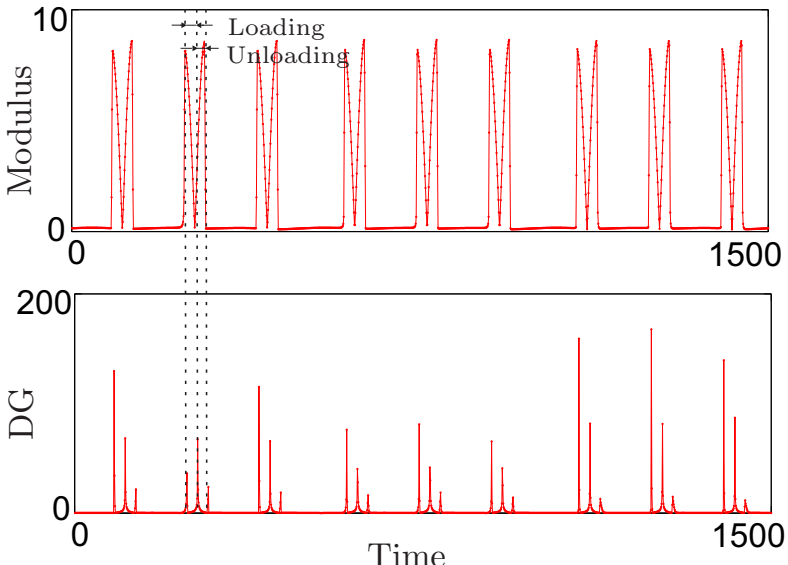

(d)
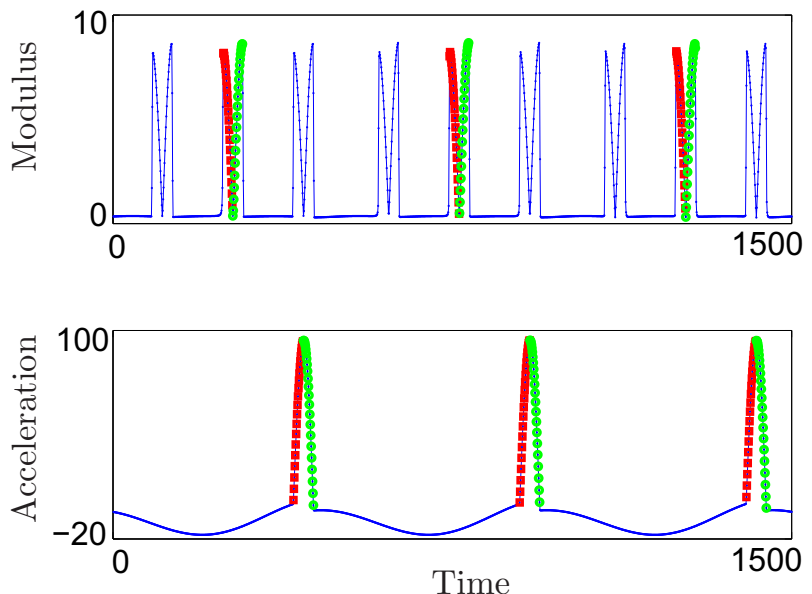

Figure 4.10: (colour online) Tangent vector analysis of the simulated data. (a) The reconstructed 3D (1st, 7th, and 13th dimensions) phase portrait with $d=26$ and $m=13$. Specifically, the dark blue points constitute the 3D phase portrait, the light blue arrows show the tangent vectors. The marked red and green tangent vectors represent the loading and unloading phases in the 7th dimension. Subplot (b) shows the time series of modulus, and the time series of direction gradient of tangent vectors. The tangent vectors depicted in subplot (a) are rearranged at the origin in subplot (c). Subplot (d) shows the result of the impact detection. The average loading duration is $5.713 \times 10^{-4} \mathrm{~s}$ per excitation period, and the average unloading duration is $5.375 \times 10^{-4}$ s per excitation period. 


\subsection{Stiffness estimation}

The results of the analytical predictions (blue curve), numerical simulations (red curve), and experiments (red circles and green dots) are displayed in Fig. 4.11. The experimental results show the same trend as these two curves. Difference between the simulation curve and the analytical curve is variable, the main difference is expected to come from the non-negligible dissipation of energy during impacts. Due to the energy dissipation, the entire impact trajectory can not complete a half circle since the separation velocity between drillbit and rock is less than their meeting velocity; while the analytical duration is predicted based on the half circle; hence, the analytical prediction is slightly larger than the corresponding simulated duration. Furthermore, the smaller the stiffness, the more the energy dissipation during impacts. This can be an explanation why their difference increases as the decreasing stiffness.

The loading stiffness estimation procedure is illustrated by using the sandstone2-indenter 1 case, which is shown in Fig. 4.11 (a). Its stable loading stiffness measured by the quasi-static rock deformation test is $21.40 \mathrm{kN} / \mathrm{mm}$. The loading duration detected from its experimental acceleration signal is $5.581 \times 10^{-4} \mathrm{~s}$. The position of $5.581 \times 10^{-4} \mathrm{~s}$ on the simulated loading curve points the abscissa $22.60 \mathrm{kN} / \mathrm{mm}$ with the estimation error $5.61 \%$. In a similar way, the loading stiffness is estimated as $23.49 \mathrm{kN} / \mathrm{mm}$ according to the analytical loading curve, with the estimation error $9.77 \%$. The same method was applied to the nine test cases, the estimation results are listed in Table 4.4. According to the simulated curves, the average estimation errors are $6.20 \pm 1.60 \%$ for the loading stiffness, and $4.78 \pm 6.17 \%$ for the unloading stiffness. When the analytical curves are used for estimation, the average estimation errors are $5.16 \pm 6.09 \%$ for the loading stiffness, and $7.18 \pm 4.28 \%$ for the unloading stiffness.

(a)

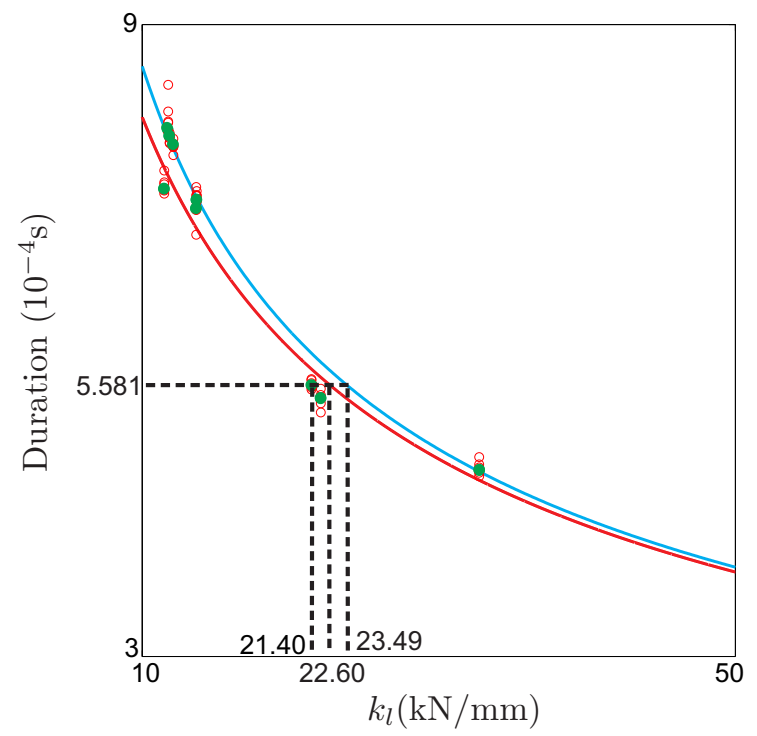

(b)

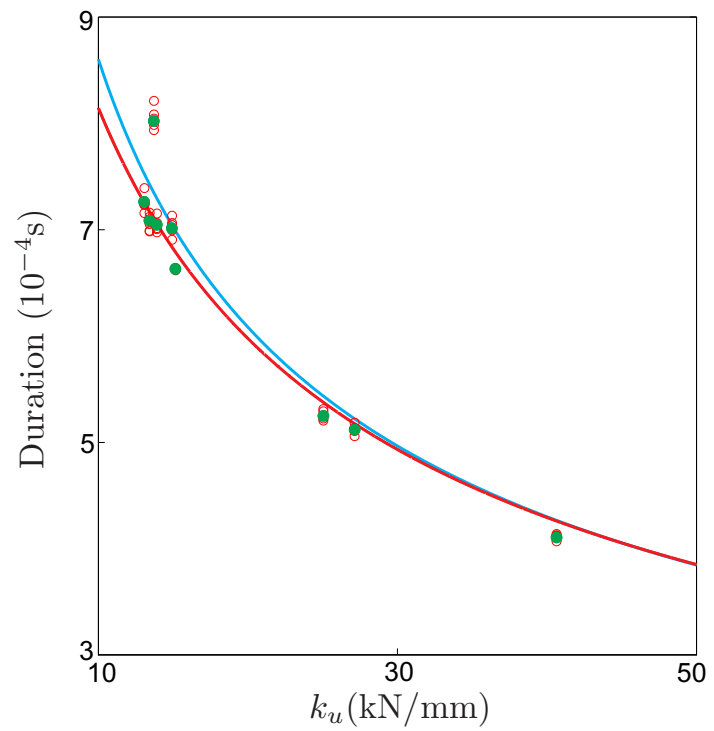

Figure 4.11: (colour online) Stiffness estimation. Subplots (a) and (b) show the estimations of the loading and unloading stiffnesses, respectively. In each plot, the blue curve represents the analytical prediction, and the red curve is the result of numerical simulation. The red circles show the detected impact durations from the collected experimental signals. For each experimental case, the average impact duration is calculated, and is represented by a green dot. An example of the loading stiffness estimation is illustrated in subplot (a). For this case, the measured stable loading stiffness is $21.40 \mathrm{kN} / \mathrm{mm}$, and the detected loading duration is $5.581 \times 10^{-4} \mathrm{~s}$. Based on the simulated curve, the loading stiffness is estimated as $22.60 \mathrm{kN} / \mathrm{mm}$. According to the analytical curve, the loading stiffness is estimated as $23.49 \mathrm{kN} / \mathrm{mm}$. 
Table 4.4: Results of the estimated stiffnesses and the corresponding errors. The loading and unloading stiffnesses were estimated based on both the numerical simulation curves and analytical prediction curves. Relative to the stable stiffnesses measured by quasi-static rock deformation tests, the corresponding estimation errors were calculated.

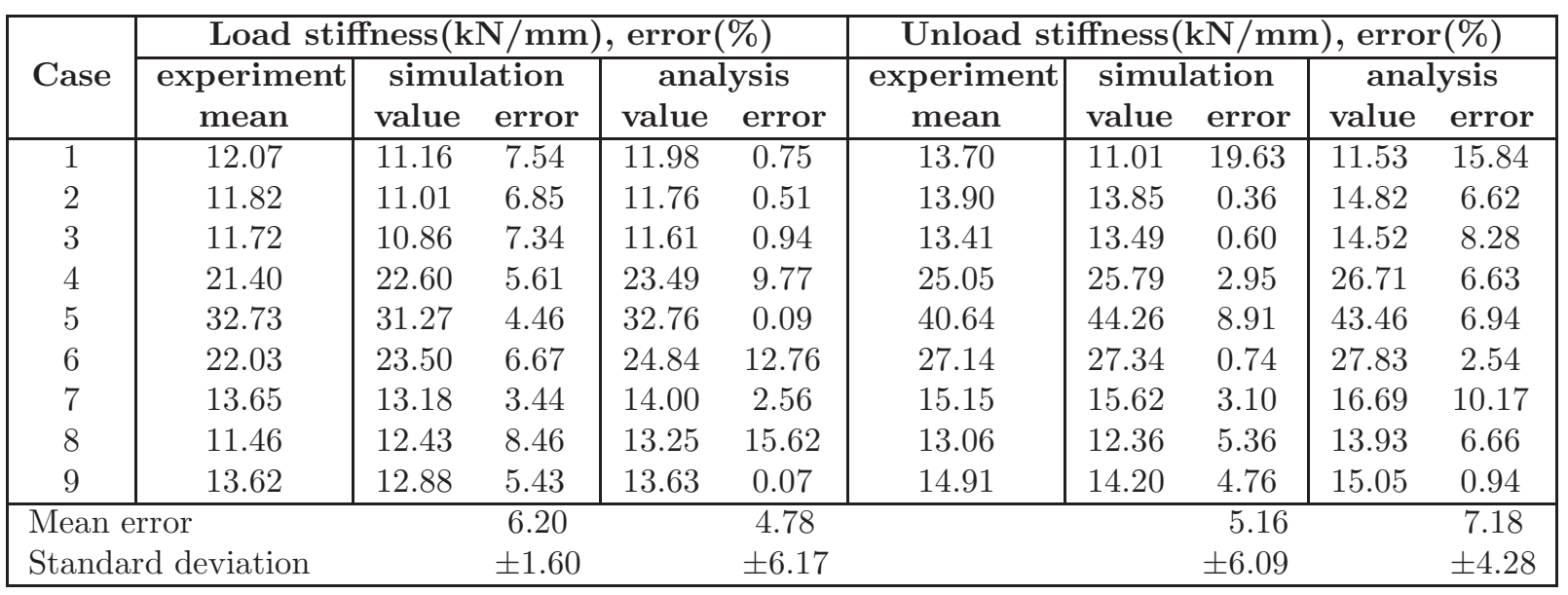

\section{Concluding remarks}

In the present work, the dynamical stiffness of an impacted object was estimated according to the developed stiffness-impact duration correlation. Since the impact duration can be detected in real-time from the acceleration time history, this offers the potential for real-time control and tuning of the optimal drilling parameters as formation properties change.

In this paper, nonlinear time series methods were used to detect impacts from simulated and experimental time series. Through analyses of modulus and direction gradients of tangent vectors of reconstructed 3D phase portraits, the rates of both the change of size and the change of direction of tangent vectors were obtained, and the peaks of their time series indicate impacts. The stiffness identification was introduced based on a 1-DOF impact oscillator system and a $2 \frac{1}{2}$-DOF rock impacting system. Comparing these two systems, the second one further developed the rock contact law and the vibration of the components above the drillbit; hence, the second system is closer to the actual percussive drilling. For both systems, the analytical predictions of their impact durations in period-one one-impact motions were developed, the corresponding numerical simulations and experiments were carried out to verify the analytical predictions. According to the analytical prediction, the impact duration was half of the natural period of the impact mass-impacted object system. Hence, when the impact mass was not changed, the impact duration decreased as the increase of the stiffness of the impacted object. This monotone correlation was utilised reversely to estimate the stiffness of the impacted object according to the detected impact duration. For the experimental cases discussed, the errors of the stiffness estimations remained relatively small. This verified the proposed method of stiffness identification.

As mentioned in the beginning, the present work can have benefits for percussive drilling applications. Specifically, the interaction between a drillbit and different formations will cause different impact durations under the period-one one-impact motions. In a similar way as presented in this paper, the correlation between the stiffness of rock and the impact duration can be developed by analytical prediction and/or numerical simulation. During the drilling process, the impact duration detected via nonlinear time series analysis and tangent vector analysis of the collected dynamic signal can infer the stiffness of the drilled rock. The identified rock stiffness will guide the adjustment of percussive drilling parameters. 


\section{Appendix A. Physical Model and Equations of Motion}

(a)

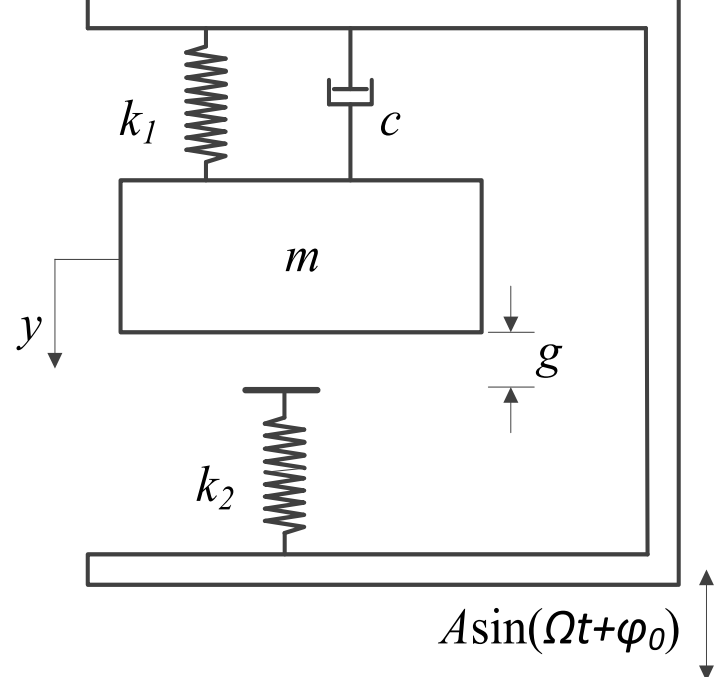

(b)

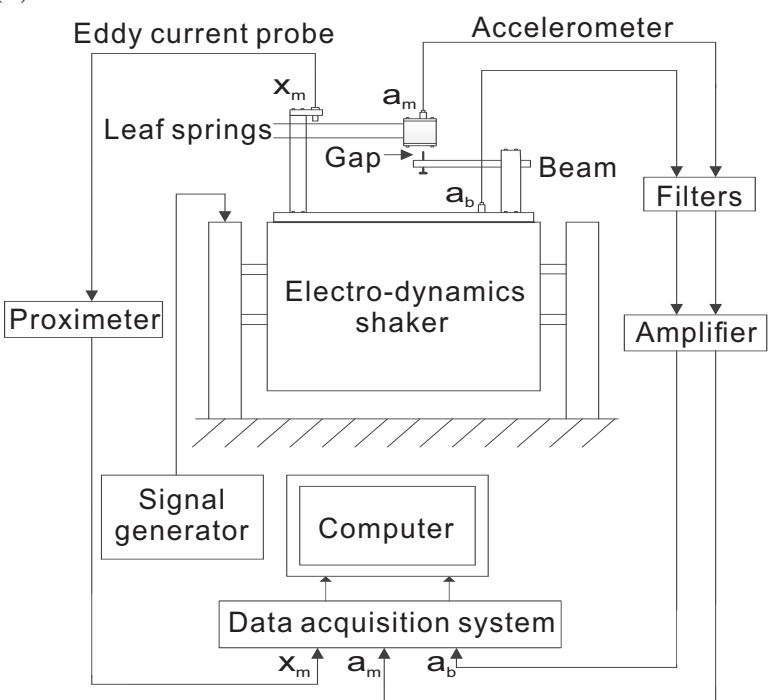

Figure A.1: (a) is the physical model of the impact oscillator. Specifically, $m$ is a block, $c$ is a damper, $k_{1}, k_{2}$ represent two springs, and $g$ is the gap. $A\left(\sin \Omega t+\varphi_{0}\right)$ indicates the external excitation, while $y$ represents the relative displacement. While (b) is the corresponding schematic of experimental set-up, whose core part is the impact oscillator.

The physical model of the impact oscillator investigated in the present article is shown in Fig. A.1 (a). The model is comprised of a mass $m$ connected to a frame via a linear spring $k_{1}$ and a damper c. A secondary linear spring $k_{2}$ is attached to the frame at a distance $g$ from the equilibrium position of the mass. The frame is subject to an external harmonic excitation, in such a way that when the amplitude of the mass oscillation is larger than the gap $g$, the mass impacts the secondary spring and hence the restoring force is governed by the overall stiffness $k_{1}+k_{2}$. During operation, the difference $y-g$ is monitored so as to detect an impact between the mass and the secondary spring. Thus, the system can operate under one of two modes at any time: no impact or impact with the secondary spring. The nondimensional equations of motion of the impact oscillator can be written in compact form as follows $[41,44]$ :

$$
\left\{\begin{array}{l}
x^{\prime}=v, \\
v^{\prime}=a \omega^{2} \sin \left(\omega \tau+\varphi_{0}\right)-2 \xi v-x-\beta(x-e) H(x-e),
\end{array}\right.
$$

where $H(\cdot)$ stands for the Heaviside step function and $x^{\prime}, v^{\prime}$ denote differentiation with respect to the nondimensional time $\tau$. In Eq. (A.1), the variables and parameters of the system have been nondimensionalized as follows:

$$
\begin{aligned}
& \omega_{n}=\sqrt{\frac{k_{1}}{m}}, \quad \tau=\omega_{n} t, \quad \omega=\frac{\Omega}{\omega_{n}}, \quad \xi=\frac{c}{2 m \omega_{n}}, \\
& x=\frac{y}{y_{0}}, \quad e=\frac{g}{y_{0}}, \quad a=\frac{A}{y_{0}}, \quad \beta=\frac{k_{2}}{k_{1}},
\end{aligned}
$$

where $y_{0}>0$ is an arbitrary reference distance, $\omega_{n}$ is the natural angular frequency, $\omega$ is the frequency ratio, $\beta$ is the stiffness ratio, $\xi$ is the damping ratio, $a$ is the nondimensionalized forcing amplitude, and $e$ is the nondimensionalized gap. 


\section{References}

[1] Y. Melamed, A. Kiselev, M. Gelfgat, D. Dreesen, J. Blacic, "Hydraulic hammer drilling technology: Developments and capabilities," Journal of Energy Resources Technology, vol. 122, pp. 1-7, 2000.

[2] M. Wiercigroch, J. Wojewoda, A.M. Krivtsov, "Dynamics of ultrasonic percussive drilling of hard rocks," Journal of Sound and Vibration, vol. 280, pp. 739-757, 2005.

[3] L.F.P. Franca, H.I. Weber, "Experimental and numerical study of a new resonance hammer drilling model with drift," Chaos, Solitons 85 Fractals, vol. 21, pp. 789-801, 2004.

[4] L.F.P. Franca, "A bit-rock interaction model for rotary-percussive drilling," International Journal of Rock Mechanics $\&$ Mining Sciences, vol. 48, pp. 827-835, 2011.

[5] W.A. Hustrulid, C. Fairhurst, "A theoretical and experimental study of the percussive drilling of rock. Part I-theory of percussive drilling," International Journal of Rock Mechanics and Mining Science 8 Geomechanics Abstracts, vol. 8, no. 4, pp. 311-333, 1971.

[6] W.A. Hustrulid, C. Fairhurst, "A theoretical and experimental study of the percussive drilling of rock. Part II-force-penetration and specific energy determinations," International Journal of Rock Mechanics and Mining Science 86 Geomechanics Abstracts, vol. 8, no. 4, pp. 335-340, 1971.

[7] W.A. Hustrulid, C. Fairhurst, "A theoretical and experimental study of the percussive drilling of rock. Part III-experimental verification of the mathematical theory," International Journal of Rock Mechanics and Mining Science $\&$ Geomechanics Abstracts, vol. 9, no. 3, pp. 417-429, 1972.

[8] W.A. Hustrulid, C. Fairhurst, "A theoretical and experimental study of the percussive drilling of rock. Part IV-application of the model to actual percussion drilling," International Journal of Rock Mechanics and Mining Science 6 Geomechanics Abstracts, vol. 9, no. 3, pp. 431-442, 1972.

[9] B. Lundberg, "Energy transfer in percussive rock destruction-I: comparison of percussive methods," International Journal of Rock Mechanics and Mining Science 8 Geomechanics Abstracts, vol. 10, no. 5, pp. 381-399, 1973.

[10] B. Lundberg, "Energy transfer in percussive rock destruction-II: supplement on hammer drilling," International Journal of Rock Mechanics and Mining Science 8 Geomechanics Abstracts, vol. 10, no. 5, pp. 401-419, 1973.

[11] B. Lundberg, "Energy transfer in percussive rock destruction-III: stress wave transmission through joints," International Journal of Rock Mechanics and Mining Science 8 Geomechanics Abstracts, vol. 10 , no. 5, pp. 421-435, 1973.

[12] S. Powell, A. Garcia, H. Barocio, A. Alonso, "Percussion drilling system combined with hybrid PDC bit increases ROP and interval drilled on Taoudenni basin well in Mauritania," in SPE/IADC Drilling Conference and Exhibition, (London, England, UK), 2015.

[13] M. Wiercigroch, Resonance enhanced drilling: Method and apparatus. Patent No. WO2007141550, 2007.

[14] E. Pavlovskaia, D.C. Hendry, M. Wiercigroch, "Modelling of high frequency vibro-impact drilling," International Journal of Mechanical Sciences, vol. 91, pp. 110-119, 2005. 
[15] S. Bargach, I. Falconer, C. Maeso, J. Rasmus, "Real-time LWD: Logging for drilling," Oilfield Review, vol. 12 , no. 3 , pp. $58-78,2000$.

[16] M. Gearhart, K.A. Ziemer, O.M. Knight, "Mud pulse MWD systems report ," Journal of Petroleum Technology, vol. 33, no. 12, pp. 2301-2306, 1981.

[17] J. Schnitger, J.D. Macpherson, "Signal attenuation for electromagnetic telemetry systems," in SPE/IADC Drilling Conference, (Amsterdam, Netherlands), 2009.

[18] J.B. Fay, H. Fay, "Wired pipes for a high-data-rate MWD system ," in European Petroleum Conference, (Cannes, France), 1992.

[19] C. McCartney, S. Allen, M. Hernandez, D. MacFarlane, A. Baksh, M.E. Reeves, "Step-change improvements with wired-pipe telemetry," in SPE/IADC Drilling Conference and Exhibition, (Amsterdam, Netherlands), 2009.

[20] M. Wiercigroch, "Bifurcation analysis of harmonically excited linear oscillator with clearance," Chaos, Solitons \& Fractals, vol. 4, no. 2, pp. 297-303, 1994.

[21] J. Ing, E. Pavlovskaia, M. Wiercigroch, S. Banerjee, "Bifurcation analysis of an impact oscillator with a one-sided elastic constraint near grazing," Physica D: Nonlinear Phenomena, vol. 239, no. 6, pp. 312-321, 2010 .

[22] O. Makarenkov, J.S.W. Lamb, "Dynamics and bifurcations of nonsmooth systems: A survey," Physica D: Nonlinear Phenomena, vol. 241, no. 22, pp. 1826-1844, 2012.

[23] B.B. Okolewska, K. Czolczynski, T. Kapitaniak, "Hard versus soft impacts in oscillatory systems modeling," Communications in Nonlinear Science and Numerical Simulation, vol. 15, no. 5, pp. 1358$1367,2010$.

[24] E. Pavlovskaia, M. Wiercigroch, "Analytical drift reconstruction for visco-elastic impact oscillator operating in periodic and chaotic regimes," Chaos, Solitons 63 Fractals, vol. 19, pp. 151-161, 2004.

[25] J. Páez Chávez, E. Pavlovskaia, M. Wiercigroch, "Bifurcation analysis of a piecewise-linear impact oscillator with drift," Nonlinear Dynamics, vol. 77, pp. 213-227, 2014.

[26] P. Thota, H. Dankowicz, "TC-HAT: A novel toolbox for the continuation of periodic trajectories in hybrid dynamical systems," SIAM J. Appl. Dyn. Sys., vol. 7, no. 4, pp. 1283-1322, 2008.

[27] O.K. Ajibose, M. Wiercigroch, E. Pavlovskaia, A.R. Akisanya, G. Károlyi, "Drifting impact oscillator with a new model of the progression phase," Journal of Applied Mechanics, vol. 79, p. 061007, 2012.

[28] O.K. Ajibose, M. Wiercigroch, A.R. Akisanya, "Experimental studies of the resultant contact forces in drillbit-rock interaction," International Journal of Mechanical Sciences, vol. 91, pp. 3-11, 2015.

[29] E. Bradley, H. Kantz, "Nonlinear time-series analysis revisited," Chaos: An Interdisciplinary Journal of Nonlinear Science, vol. 25, p. 097610, 2015.

[30] N. Packard, J. Crutchfield, J. Farmer, R. Shaw, "Geometry from a time series," Physical Review Letters, vol. 45, no. 9, pp. 712-716, 1980. 
[31] F. Takens, "Detecting strange attractors in fluid turbulence," Lecture Notes in Mathematics, vol. 898, pp. 366-381, 1981.

[32] H. Kantz, T. Schreiber, Nonlinear time series analysis. Cambridge: Cambridge University Press, second ed., 2004.

[33] H. Hegger, H. Kantz, T. Schreiber, "Practical implementation of nonlinear time series methods: The TISEAN package," Chaos, vol. 9, pp. 413-435, 1999.

[34] A.M. Fraser, H. L. Swinney, "Independent coordinates for strange attractors from mutual information," Physical Review A, vol. 33, no. 2, pp. 1134-1140, 1986.

[35] M.B. kennel, R. Brown, H.D.I. Abarbanel, "Determining embedding dimension for phase-space reconstruction using a geometrical construction," Physical Review A, vol. 45, no. 6, pp. 3403-3411, 1992.

[36] H. Kantz, "A robust method to estimate the maximal Lyapunov exponent of a time series," Physics Letter A, vol. 185, pp. 77-87, 1994.

[37] H. Kantz, T. Schreiber, "Dimension estimates and physiological data," Chaos, vol. 5, no. 1, pp. 143$154,1995$.

[38] J.P. Eckmann, S.O. Kamphorst, D. Ruelle, "Recurrence plots of dynamical systems," Europhysics Letters, vol. 4, no. 9, pp. 973-977, 1987.

[39] V.G.S. Simionatto, H.H. Miyasato, F.M. Melo, M.D. Junior, "Singular mass matrices and half degrees of freedom: A general method for system reduction," in 21st International Congress of Mechanical Engineering, (Natal, RN, Brazil), 2011.

[40] M. Liao, J. Ing, J. Páez Chávez, M. Wiercigroch, "Bifurcation techniques for stiffness identification of an impact oscillator," Commun. Nonlinear Sci. Numer. Simul. doi: 10.1016/j.cnsns.2015.11.027.

[41] J. Ing, E. Pavlovskaia, M. Wiercigroch, S. Banerjee, "Experimental study of impact oscillator with one-sided elastic constraint," Philos. Trans. R. Soc. Lond., Ser. A, Math. Phys. Eng. Sci., vol. 366, no. 1866 , pp. $679-704,2008$.

[42] M. Sayah, Nonlinear time series analysis applied to resonance enhanced drilling. University of Aberdeen: PhD thesis, 2015.

[43] M. Sayah, M.D. Baptista, J. Ing, M. Wiercigroch, "Attractor reconstruction of an impact oscillator for parameter identification," International Journal of Mechanical Sciences, vol. 103, pp. 212-223, 2015.

[44] S. Banerjee, J. Ing, E. Pavlovskaia, M. Wiercigroch, K.R. Ramesh, "Invisible grazings and dangerous bifurcations in impacting systems: The problem of narrow-band chaos," Physical Review E Statistical, Nonlinear, and Soft Matter Physics, vol. 79, no. 3, p. 037201, 2009. 